Mon. Not. R. Astron. Soc. 000, 田?? (2001) Printed 15 February $2019 \quad$ (MN LATEX style file v1.4)

\title{
Infrared Spectroscopy of Substellar Objects in Orion
}

\author{
P.W.Lucas ${ }^{1}$, P.F.Roche ${ }^{2}$, France Allard $^{3}$ and P.H.Hauschildt ${ }^{4}$ \\ ${ }^{1}$ Dept. of Physical Sciences, University of Hertfordshire, College Lane, Hatfield AL10 9AB. \\ email:pwl@star.herts.ac.uk \\ ${ }^{2}$ Astrophysics Dept., University of Oxford, 1 Keble Road, Oxford OX1 3RH. \\ ${ }^{3}$ CRAL, Ecole Normale Superieure, 46 Allee d'Italie, Lyon, 69364 France, Cedex 07 \\ ${ }^{4}$ Dept. of Physics and Astronomy \& Centre for Simulational Physics, University of Georgia, Athens, GA 30602-2451
}

Accepted 2001 May, Received 2001 March

\begin{abstract}
We present broad band spectra of a sample of 21 low luminosity sources in the Trapezium Cluster, with masses in the range $0.008-0.10 \mathrm{M}_{\odot}$ (assuming an age of $1 \mathrm{Myr}$ ). These were selected for low extinction in most cases and are located west of the brighter nebulosity. The spectra are in the $H$ bandpass $(1.4-1.95 \mu \mathrm{m})$ and $K$ bandpass $(1.9-2.5 \mu \mathrm{m})$ also for most of the brighter sources, with a resolution of $50 \mathrm{~nm}$. They were taken with the United Kingdom Infrared Telescope (UKIRT) using the CGS4 spectrometer. Absorption by water vapour bands is detected in all the substellar candidates except one, which is a highly reddened object with strong $\mathrm{H}_{2}$ emission and an anomalously blue $(I-J)$ colour, implying that it is a very young cluster member with circumstellar matter. The observation of prominent water vapour bands confirms the low Effective Temperatures implied by our $(I-J)$ colour measurements in an earlier paper and would imply late $\mathrm{M}$ or L spectral types if these were older field dwarfs. However, the profiles of the $H$ bandpass spectra are very different from those of field dwarfs with similar water absorption strength, demonstrating that they are not foreground or background objects. In addition, the CO absorption bands at $2.3 \mu \mathrm{m}$ and the NaI absorption feature at $2.21 \mu \mathrm{m}$ are very weak for such cool sources. All these features are quite well reproduced by the AMES-Dusty-1999 model atmospheres of Allard et al.(2000,2001), and arise from the much lower gravities predicted for the Trapezium sources $(3.5<\log (g)<4.0)$ compared to evolved objects $(\log g \sim 5.5)$, This represents a new proof of the substellar status of our sources, independent of the statistical arguments for low contamination, which are reexamined here. The very late spectral types of the planetary mass objects and very low mass brown dwarfs demonstrate that they are cluster members, since they are too luminous to be field dwarfs in the background. We also present additional UKIRT photometry of a small region in the south of the Trapezium cluster where the extinction and nebular brightness are low, which permitted the detection of objects with $1 \mathrm{Myr}$ masses slightly lower than our previous least massive source at $8 \mathrm{M}_{J u p}$. Following a minor update to our previous $J$ band photometry, due to a new UKIRT filter calibration, there are $\sim 15$ planetary mass candidates in the full dataset.
\end{abstract}

Key words: stars:low-mass,brown dwarfs, stars: pre-main sequence, stars: formation, (stars:) circumstellar matter

\section{INTRODUCTION}

Brown dwarf candidates have been discovered in large numbers in many star formation regions and very young stellar clusters, through deep infrared photometric searches (eg. Comeron et al. 1993,1996; McCaughrean et al. 1995; Bejar, Zapatero-Osorio \& Rebolo 1999). Some of these surveys have yielded objects of planetary mass, in the Chamaeleon I molecular cloud (Tamura et al.1998; Oasa et al.1999) in the $\sigma$-Orionis cluster (Zapatero-Osorio et al.2000) and in the Trapezium Cluster in Orion (Lucas \& Roche 2000, hereafter Paper I; Hillenbrand \& Carpenter 2000). In most cases there is a very high probability that photometric candidates are indeed substellar cluster members. Some candidates with near infrared $J H K$ photometry exhibit $K$ bandpass excesses due to hot circumstellar dust, which demonstrate their youth

(C) 2001 RAS 
(eg. Oasa et al.1999), although this criterion will miss the majority of young stellar objects (YSOs). In the Trapezium cluster, even substellar candidates without $K$ bandpass excesses can generally be assumed to be cluster members because any measured extinction precludes confusion with foreground field dwarfs while the dense backdrop of the OMC-1 cloud and the high galactic latitude $\left(\mathrm{b}=18^{\circ}\right)$ both mitigate against background contamination.

It is still important to observe brown dwarf candidates spectroscopically, both to confirm their status and to explore the rich physics of their atmospheres, which have much lower gravities than their evolved counterparts. There are effects specific to very young sources which complicate the interpretation of their spectra. These are: (1) light scattered and emitted by circumstellar matter, which modifies the signal from the photosphere; (2) reddening by the cluster medium, which can only be imperfectly removed; (3) the lower gravity of the atmosphere. The low gravities mean that the spectra cannot be simply compared to those of Class V dwarfs and such spectra have not been successfully modelled in the near infrared until now. However, the new AMES-Dusty-1999 models of Allard et al.(2000, 2001) which employ the NASA Ames water line list and a more detailed treatment of dust, appear to be considerably more successful than previous models (see Section 4). Evidence for low gravity spectral features has been seen in the Pleiades (Martin, Rebolo \& Zapatero-Osorio 1996) which have an age of $\sim 100$ Myr. Luhman \& Rieke (1998) and Luhman et al.(1998a) published optical spectroscopy of large samples of YSOs in IC348 and L1495E, including some substellar objects. They allowed for the effects of low gravity by fitting spectra to templates formed from the average of Class $\mathrm{V}$ dwarfs and Class III giants, which produced a good match to the data. However this approach is not appropriate for the very low mass sources in the Trapezium and $\sigma$-Orionis, since the few good quality infrared spectra of late-M type Class III and Class I giants in the literature (eg. Lancon \& Rocca-Volmerange 1992) do not have similar profiles to those presented here, and correspond to even lower gravity objects $(-2<\log (g)<1$. Moreover there are no L-type giants to compare with the cooler sources found in Orion.

Wilking, Greene \& Meyer (1999) analysed $K$ bandpass spectra of brown dwarfs in $\rho$ Ophiuchus by comparison with Class V dwarfs, which may lead to errors in the derived effective temperatures. We experienced the same problem in $\mathrm{Pa}-$ per I when deriving temperatures and luminosities by comparison with Class V IJH colours. Fortuitously this does not appear to have caused significant errors because: (a) many sources with low extinction were observed with $(J-H)=0.5$ to 0.6 , indicating that this colour does not increase to Red Giant Branch values $((J-H) \sim 0.9)$ in very young brown dwarfs and (b) the AMES-Dusty-1999 models indicate that low gravity causes only small changes in the broad band colours, comparable to our typical photometric uncertainty of 0.1 magnitudes.

In this paper we present low resolution $H$ and $K$ bandpass spectra of a sample of Trapezium cluster members including low mass stars, brown dwarfs and planetary mass objects. The spectra are analysed by comparison with both the AMES-Dusty-1999 models and published spectra of Class V dwarfs. Optical spectra will be presented in a future paper. New photometric data are also presented covering the south- ern region of the Trapezium cluster. We also readdress the arguments concerning the nature of these sources, in light of the spectroscopic data.

\subsection{Note on Nomencalature}

In this paper we use the less controversial but not very descriptive term 'planetary mass objects' to describe sources below the deuterium burning threshold. Some in the extrasolar planets community object to the term 'free floating planets' to describe objects which are not orbiting a star and probably form in a molecular cloud core in a manner similar to stars (although the word planet originally meant 'wanderer'). As a possible alternative, we suggest the more compact term 'planetar' as a contraction of 'planetary mass object believed to have formed like a star'. A large amount of theoretical work is now in progress, examining a variety of different formation scenarios. There may prove to be more than one mode of formation for these low mass bodies, as is believed to be the case for stars.

\section{OBSERVATIONS}

\subsection{Spectroscopy}

Long slit spectroscopy of sources in the Trapezium cluster was carried out at the United Kingdom Infrared Telescope (UKIRT) on 27-30 November 1999, using CGS4, a near infrared Cooled Grating Spectrograph. The observer was PWL. Sources were observed in pairs aligned along the slit of the spectrograph and the sample was selected from the dataset of Paper I and the new imaging data presented here, with the intention of sampling the mass range from 0.008 to $0.10 \mathrm{M}_{\odot}$, i.e. from planetary mass to very low mass stars. 21 such sources were observed and 2 sources of higher mass. There were the following additional criteria. (1) Location in a region of low nebular surface brightness in order to improve sensitivity. (2) Low extinction towards at least one member of a pair, defined by $(J-H)<1.5$ or $\mathrm{A}(\mathrm{V}) \lesssim 7.5$, also to improve sensitivity. (3) Most sources were selected for 'normal' $(I-J)$ colours in an attempt to minimise contamination by light scattered from circumstellar matter (see Paper I) but 2 sources with the anomalously blue colours shared by $14 \%$ of the cluster population were observed to see whether their spectra were different from the others. (4) Location within $\sim 40^{\prime \prime}$ of a sufficiently bright $\operatorname{star}(J \mathrm{mag}<15)$ to permit precise location of optically invisible targets within the slit by offsetting to the target. In practice, all 21 of the low mass targets were located at least 105 arcsec west of $\theta_{1}$ Orionis $\mathrm{C}$ at the cluster centre.

CGS4 has a $256 \times 256 \mathrm{InSb}$ array sensitive from $1-5.5 \mu \mathrm{m}$ and a spatial scale of $0.62 \mathrm{arcsec} / \mathrm{pixel}$. The $40 \mathrm{l} / \mathrm{mm}$ grating was used in first order, which yields a spectral dispersion of $25 \mathrm{~nm} /$ pixel but a $1.2^{\prime \prime}$ wide slit was employed in most cases, yielding an instrumental profile with a width of $50 \mathrm{~nm}$. This corresponds to a spectral resolution of $\mathrm{R}=330$ in the $H$ bandpass and $\mathrm{R}=440$ at $K$. The wide slit was used to ensure that most of the flux from both members of a widely separated pair would fall in the slit. Each spectrum covers a $0.62 \mu \mathrm{m}$ wavelength range. The $H$ bandpass spectra were centred at $1.65 \mu \mathrm{m}$ and the $K$ bandpass spectra at $2.2 \mu \mathrm{m}$, overlapping 


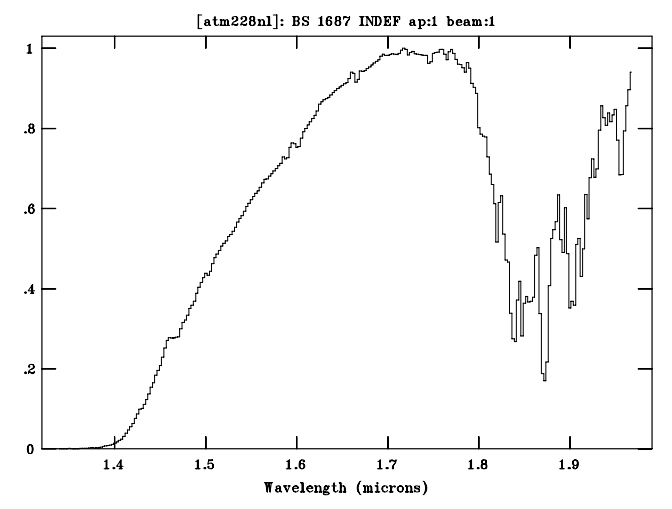

Figure 1. Relative transmission of Atmosphere and Spectrograph per unit wavelength.

in the $1.9 \mu \mathrm{m}$ region. One pair of sources was observed at $25 \mathrm{~nm}$ resolution with a $0.6^{\prime \prime}$ slit, though not in very good conditions; no additional narrow line features were revealed. This pair of sources was observed with $H$ bandpass spectra centred at $1.58 \mu \mathrm{m}$ on the first night, in attempt to include the steep $J$ band water edge at $1.34 \mu \mathrm{m}$, but the instrument efficiency in this configuration was found to be essentially zero for wavelengths $\lambda<1.4 \mu \mathrm{m}$, so the longer wavelength setting was used thereafter. The relative throughput of the instrument and the atmosphere in the $H$ bandpass is shown in Figure 1. The observations and source notes are detailed in Table 1. For all other pairs, the array was stepped over 2 pixels in a whole pixel step to remove bad pixels and the telescope was nodded along the slit in order to subtract the background, with background-limited individual integrations of 30 to $60 \mathrm{~s}$ duration. Total exposures time ranged from 10 minutes for the brightest sources to 1.5 hours for the faintest. 21 primary sources were observed in the $H$ bandpass and 11 of these in the $K$ bandpass also. We also present $H$ bandpass spectra of 2 fairly low mass sources which appeared in the long slit by chance. The smaller number of $K$ bandpass observations was due to constraints on observing time (weather and computer failures). Priority was given to $H$ bandpass observations because of anticipated complications owing to emission by hot dust in the $K$ bandpass and because the water absorption troughs are slightly more prominent in the $H$ bandpass. A small number of red main sequence stars were also observed in order to check the reliability of the data. Their spectra agree very well with those published by Leggett et al.(2000) for the same objects, also obtained with CGS4 at UKIRT.

The data were coadded into background subtracted 'reduced groups' using the CGS4dr software package and spectra were optimally extracted using the TWODSPEC package in IRAF, the Image Reduction and Analysis Facility. Argon arc spectra, tungsten lamp flatfields and standard stars were observed frequently to compensate for changes in the wavelength calibration when the slit orientation was changed and to accurately correct for the changing telluric water vapour column density. F-type stellar standards were generally used in preference to A-types since they have weaker HI absorption lines. The fairly weak Brackett series lines were removed from the stellar standards by fitting the continuum with a high order cubic spline, dividing the fit by the data to produce a line spectrum, and multiplying the data by the line spectrum. The strong Paschen $\alpha$ line at $1.875 \mu \mathrm{m}$ coincides with a deep trough due to telluric water absorption and could not be adequately removed, so we have removed a small section of the spectra near this wavelength. The Brackett series limit occurs at $1.46 \mu \mathrm{m}$ and coincides with a narrow telluric feature at the short wavelength edge of the $H$ bandpass, making it impossible to accurately remove. Inspection of the NextGen models of Hauschildt et al.(1997) indicates that the Brackett series limit is weaker than the telluric feature in F-type standards and it does not appear to be noticeable in the spectra presented here.

In addition to background shot-noise, a significant source of noise arises from the spatial gradients in the nebular surface brightness, which make the background subtraction difficult. Owing to these gradients the reduced group images are not flat, but show the effects of subtracting two frames separated by distance of the nod along the sky: either 10 or 20 arcsec was used depending on the separation between the members of a pair. The images also show the spectral residuals due to fluctuations in the strength of telluric $\mathrm{OH}$ emission lines. A polynomial was interactively fitted to the residual background at each wavelength and subtracted, using the APALL package in TWODSPEC, and this procedure was generally very successful in removing both nebulosity gradients and residual $\mathrm{OH}$ emission. The problem was greatest for the fainter sources, whose peak brightness is comparable to fluctuations in nebular suface brightness on a scale of a few arcsec, even for sources selected in regions of low nebular background. Where necessary, 2 iterations were used to produce a flat background. For the faintest sources, the background may be slightly over or under subtracted, but agreement between spectra taken at the two nod positions was used as a check to prevent this occuring. Only one spectrum was lost due to this problem: the $K$ bandpass spectrum of the faintest source, 051-147, was rendered useless by a small step in the surface brightness on the same scale as the nod length, but an $H$ bandpass spectrum was successfully extracted. We recommend that a nod length of 5 arcsec or less be used in star formation regions when taking long slit spectra of faint sources.

\subsection{Photometry}

Additional photometry in the $I J H$ bands was obtained at UKIRT, using the infrared camera UFTI, in the manner described in Paper I, using 900 s exposures. The new data were taken on the nights of 11-12 November 1999 by observatory staff. They consisted of three contiguous 1.5 arcmin UFTI fields located 3 arcmin south of $\theta_{1}$ Ori C, oriented east-west as shown in Figure 2. This region was selected because our existing data indicated that the nebular surface brightness and average extinction would be low in this region, and published $K$ bandpass maps showed that the stellar density is reasonably high (Hillenbrand 1997). These features assisted in the detection of some new very faint sources, as described in Section 3.1. The seeing conditions were fairly good, with a FWHM of $0.5-0.6^{\prime \prime}$ in all three filters, aided by the tip/tilt image stabilisation of the UKIRT secondary mirror.

Photometry was performed using the DAOPHOT crowded field photometry package in IRAF as in Paper I, using both manual photometry and automated crowded field 


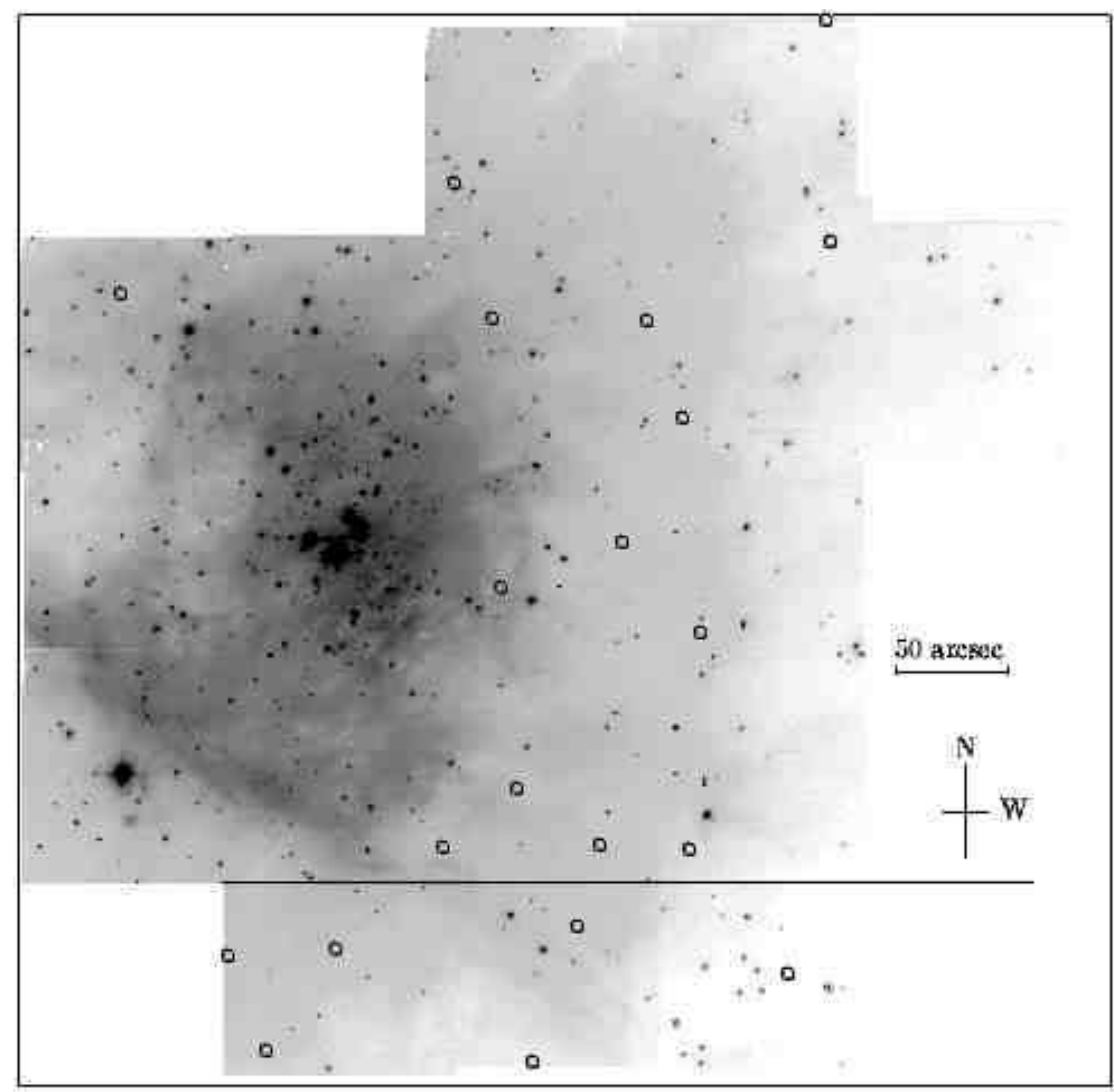

Figure 2: $J$ Bandpass image of the full photometric dataset. The region of new data is below the horizontal line. The spectroscopic sample was taken from sources to the west of the brightest nebulosity. The locations of very low mass candidates (dereddened mag $\mathrm{J}_{d r}>17.5$, or $\left.\mathrm{M} \lesssim 0.016 \mathrm{M}_{\odot}\right)$ are marked with circles.

photometry. The former was generally preferred in order to accurately subtract the highly structured nebulous background, except when the image profiles of sources overlapped significantly. Several sources were contained in the overlap region between the new data and the dataset of Paper I. The measured magnitudes typically differed by $0.1 \mathrm{mag}$ or less, which is approximately the photometric error described in Paper I. The main source of error in the brighter sources is the image profiles, which are spatially variable in the overlap region since this is the very edge of both the old and new datasets. Each UFTI field is made up of a 9-point image mosaic with small jitters.

\subsubsection{Calibration Update}

The $J$ band magnitude system for UFTI at UKIRT has recently been updated by observatory staff, who discovered that the transformations from the old IRCAM system to the new UFTI system are different for cool red stars than other main sequence stars. This is because these stars have water absorption bands in similar locations to the water bands in the earth's atmosphere and UFTI employs the new $J$ band filter developed for the Mauna Kea Consortium, optimised for the low water column above this observing site. The new transformation has been thoroughly calibrated via measurements of late M type and L type stars. Since our Paper I dataset was calibrated using red stars (in order to calculate transformations from Cousins $I$ to the UFTI $I$ band) this requires us to update our $J$ band photometry, and consequently the I band transformations also (see UKIRT web pages for the details). The new $J$ band magnitudes are typically 0.1 magnitudes brighter than before, which leads to a slightly lower luminosity for sources with significant extinction, since the calculated extinction is reduced. Sources with near zero extinction are slightly more luminous than had been thought. However changes of this order are of very little consequence, since the mass-luminosity relation is quite steep throughout the brown dwarf regime and the change is similar to both the typical photometric uncertainty and the theoretical uncertainty in the mass-luminosity conversion.

The bolometric correction, $B C_{J}$, formerly used to convert dereddened $J$ magnitudes, $J_{d r}$, to luminosities is also updated in consequence:

$$
\begin{array}{r}
B C_{J}=2.01 ; J_{d r} \geq 13.71 \\
. B C_{J}=0.209 J_{d r}-0.855 ; J_{d r}<13.71
\end{array}
$$

The new models used here predict the fluxes in each filter directly without the need for a bolometric correction.

\section{RESULTS}




\subsection{Photometry}

43 new sources were detected at both $J$ and $H$ bands in the new data, of which 31 were detected at $I$ band. A colourluminosity plot, with the new data added to that of Paper I, is shown in Figure 3 for all 557 unsaturated sources. We plot 2 different $1 \mathrm{Myr}$ isochrones as almost vertical solid lines at the left of the picture, to which sources may be dereddened. Both of these are self consistent predictions using the same model atmospheres for colour predictions and to provide the boundary condition for the evolutionary calculations. This removes the need to use uncertain bolometric corrections. Furthest to the left is an isochrone supplied by Baraffe et al.(2001, in preparation), using the non-gray AMES-Dusty-1999 model atmospheres (Allard et al.2000, 2001). The isochrone slightly to the right is the prediction of Baraffe et al.(1998), recently extended to 1 Myr and to the deuterium burning threshold (Chabrier et al.2000). This second isochrone has evolution and colours based upon the NextGen model atmospheres. It is useful to see both because the Nextgen atmospheres correctly predict the colours of main sequence stars with effective temperatures above $2500 \mathrm{~K}$, whereas the AMES-Dusty models are successfully fit the colours of field brown dwarfs, below $2200 \mathrm{~K}$, where dust has an important influence on the photosphere. The temperature at the deuterium burning threshold lies near the low temperature end of the NextGen regime but we cannot be sure which model will predict the $(J-H)$ colours better in very young, low gravity photospheres. The true colour probably lies between the 2 predictions, as discussed below.

Recalibrated photometry of the full dataset is available electronically 円. The O'Dell \& Wong (1996) coordinate based naming system is used as in Paper I. The advantageous features of the new dataset, as described above, permitted the detection of some very low luminosity sources: 4 new planetary mass candidates, 3 of which appear to be less massive than any of the sources described in Paper I, assuming an age of $1 \mathrm{Myr}$ and employing either the isochrones of Burrows et al.(1997, hereafter BM97) or the new isochrone of Baraffe et al. However only 1 of these 3 faint sources has good photometry: 189-659 has $m_{J}=19.23,(J-H)=0.63$, which indicates a notional mass of $6.2 \mathrm{M}_{J u p}$ at $1 \mathrm{Myr}$ (on the BM97 isochrone) and apparently negligible extinction. The other 2 sources have large photometric uncertainties in both the $J$ and $H$ bands, so their masses are highly uncertain. Intriguingly, these 3 faint sources are all located close together on the sky, in a 1 arcmin square region 3 arcmin due south of $\theta_{1}$ Ori $\mathrm{C}$, on the far side of the Orion Bar from the cluster centre. The Orion Bar is the ionisation front of the OMC-1 cloud, viewed edge-on as it curves into the line of sight. Sources which are located beyond it lie on lines of sight passing deep into the molecular cloud. Hence we might expect such sources to be younger than the rest of the cluster and hence less massive for a given luminosity. However, the very low extinction toward 189-659 indicates that this source probably lies in front of the cloud and might possibly be a minimum mass star in the foreground. The locations of all the sources with dereddened $J$ magnitudes $J_{d r} \geq 17.5$

* Photometry and spectra are available via anonymous FTP to star.herts.ac.uk, in pub/Lucas/Orion.

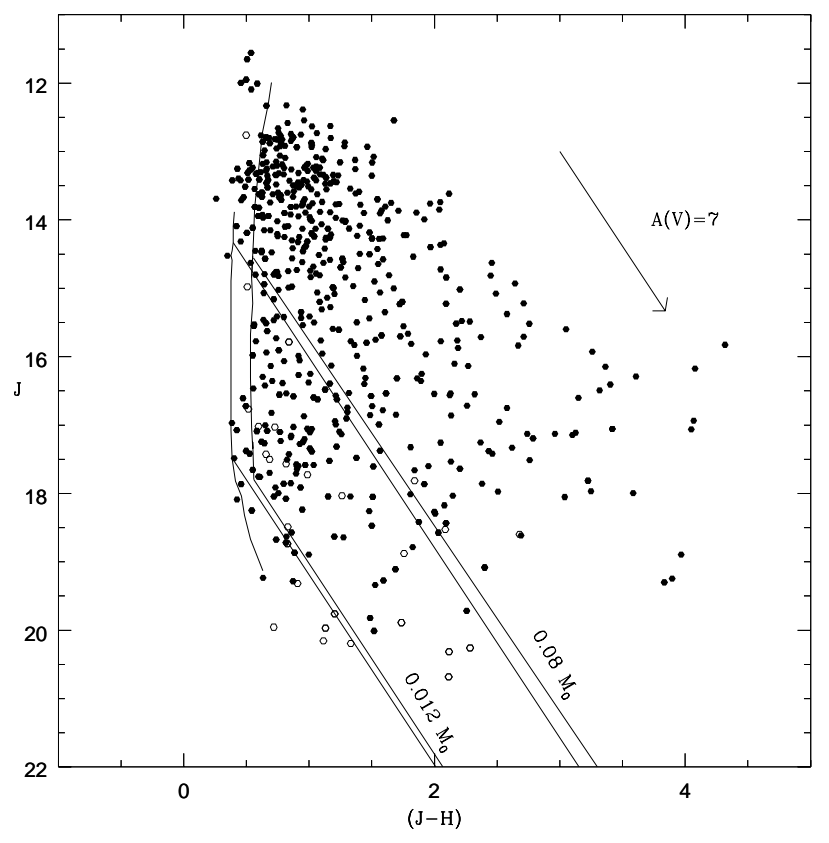

Figure 3: Colour-magnitude diagram for the 557 unsaturated $J$ bandpass sources. Two theoretical $1 \mathrm{Myr}$ isochrones are plotted at left, with reddening lines indicated for $0.08 \mathrm{M}_{\odot}$ and the $0.012 \mathrm{M}_{\odot}$ deuterium threshold. Open circles represent sources with large $J$ band photometric uncertainties $(\geq 0.2 \mathrm{mag})$.

(i.e. $\mathrm{M} \leq 0.012$ to $0.016 \mathrm{M}_{\odot}$ at $1 \mathrm{Myr}$ ) are plotted in Figure 2 .

There are now approximately 15 planetary mass candidates in the dataset, following the filter recalibration. The number of sources below the deuterium burning threshold cannot be specified very precisely, mainly because of the uncertainty in the $(J-H)$ colour of their photospheres mentioned earlier. Most evolutionary calculations are in very good agreement concerning the minimum mass for deuterium burning, at 0.012-0.013 $\mathrm{M}_{\odot}$ (Saumon et al.1996; BM97; Chabrier et al.2000). In addition, the $1 \mathrm{Myr}$ isochrones of BM97, D'Antona \& Mazzitelli (1998) (herafter DM98, update to 1997 publication) and Baraffe et al.(1998) are in fair agreement (within 0.2 to $0.3 \mathrm{mag}$ ) with regard to source luminosities for low mass brown dwarfs. However, the small uncertainty in the $(J-H)$ colours can slightly change the number of planetary mass objects by moving the lines of constant mass in Figure 3 to the right or to the left by $0.1-0.15 \mathrm{mag}$, since a number of sources appear to lie very close to the deuterium burning threshold. Applying the new Baraffe et al. isochrone, there are $\sim 13$ planetary mass objects (below the $\mathrm{M}<0.012 \mathrm{M}_{\odot}$ threshold calculated by Chabrier et al.), whereas the NextGen-based Baraffe et al. ischrone indicates there are 16 such objects. Using the main sequence colours and bolometric corrections as in Paper I, and the BM97 isochrone for luminosity, indicates that there are 15 such objects. Given that the $J$ bandpass photometric errors are typically 0.1-0.2 mag for these faint candidates, 
the important issue is not the precise number but whether the IMF extends below the deuterium burning threshold, which appears to be the case.

The NextGen colour predictions, using the MillerTennyson water line list, are only very slightly too red for main sequence stars but these atmospheres poorly reproduce the observed profiles of both field dwarfs with strong water absorption (Leggett et al. 2001) and the low gravity spectra presented here. The AMES-Dusty-1999 colour predictions, using the NASA AMES water line list (Partridge \& Schwenke 1997), are 0.1 to $0.2 \mathrm{mag}$ too blue in main sequence stars (see Allard, Hauschildt \& Schwenke 2000) but reproduce the profiles of the spectra presented here quite well. A small colour discrepancy in $(J-H)$ would appear to arise from incompleteness in the water line list in the 1.7$2.2 \mu \mathrm{m}$ water absorption band, which is shown directly for Trapezium sources in Section 4.1.

Note that we have made a conservative choice of reddening law (see Paper I). The choice has little effect in any case because the planetary mass candidates necessarily have low extinction and because extinction laws have little variation in the infrared (see Cardelli, Clayton \& Mathis (1989)) except in extremely dense environments such as the accretion disks of embedded protostars, which may contain dust grains larger than $1 \mu \mathrm{m}$ in radius.

There may be small but unquantified effects on the $(J$ $H$ ) colours due to scattering by circumstellar matter and a small percentage of emission by hot circumstellar dust in some cases. Hot dust would make sources appear slightly redder (see Section 4) and overmassive; the direction of any scattering effect depends very much on assumptions about the circumstellar structure and viewing angle. The additional uncertainties of cluster membership and candidate ages are discussed in Section 6.

Erratum

We note that 1 planetary mass candidate from Paper I was found to be double counted owing to a typing error. An internal cross check of all the coordinates shows that this is the only such case in the dataset of 557 unsaturated sources detected in the $J$ and $H$ bandpasses.

\subsection{Spectroscopy}

\subsection{1 $H$ band}

$21 H$ band spectra were taken of sources with masses $\mathrm{M}$ $\lesssim 0.1 \mathrm{M}_{\odot}$. These are plotted in Figure $4(\mathrm{a}-\mathrm{c})$, as $\mathrm{F}_{\nu}$ spectra in order to suppress the apparent noise at short wavelengths. Dereddened spectra are plotted above the observed spectra for those sources with non-zero extinction. These spectra are not flux calibrated since for some observations observing conditions were not photometric. The fainter sources are Gaussian-smoothed to enhance low resolution features. Nearly all of the spectra are dominated by strong water absorption bands to either side of a sharp peak located between 1.68 and $1.70 \mu \mathrm{m}$. The exceptions are plotted in the lower part of Figure 4(b). No real narrow spectral features are unambiguously detected in the $H$ bandpass. Pixel to pixel variations therefore illustrate the noise in each spectrum, which has wavelength dependent contributions from narrow telluric $\mathrm{OH}$ emission lines and broad band telluric water absorption, both of which vary on a timescale of minutes during an exposure. The noise is most obvious in very faint sources such as 084-104 (Figure 4(c)) in which many spurious narrow features remain despite some smoothing. The data quality is poor in the 1.8-1.95 $\mu \mathrm{m}$ region owing to strong telluric water absorption and also becomes less reliable at $\lambda<1.5 \mu \mathrm{m}$, due to both telluric water absorption and the declining instument efficiency. Some of the spectra are strongly reddened, with the result that the water absorption appears weaker on the long wavelength side of the peak and stronger on the short wavelength side, eg. object 013-306. In most cases the effect is minor owing to our preferential selection of sources with low extinction. The extinction toward each source is calculated using the $I J H$ photometry, by comparison with measured main sequence star colours as described in Paper I, and noted in Figure 4(a-c). The DEREDDEN task in IRAF was used, which is based on the Cardelli, Clayton \& Mathis extinction law, with reddening parameter $\mathrm{R}_{V}=5.5$ as before and $\mathrm{A}(\lambda) \propto \lambda^{-1.61}$ in the infrared. This is a somewhat imprecise procedure, because our typical photometric uncertainty of $0.1 \mathrm{mag}$ in each filter leads to an uncertainty of $0.14 \mathrm{mag}$ in the $(J-H)$ colour, which corresponds to an uncertainty of $0.25 \mathrm{mag}$ in $H$ band extinction and $1.2 \mathrm{mag}$ in $V$ band extinction. However, the errors will be less for the brighter sources in the region of the spectroscopic sample, which was selected for faint nebulous background. The use of main sequence colours gives similar results to the NextGen atmospheres: the atmospheric models predict changes of no more than $\sim 0.1 \mathrm{mag}$ in $(J-H)$ due to lower gravity in young sources, so we do not think this uncertainty will be larger than measurement error. 

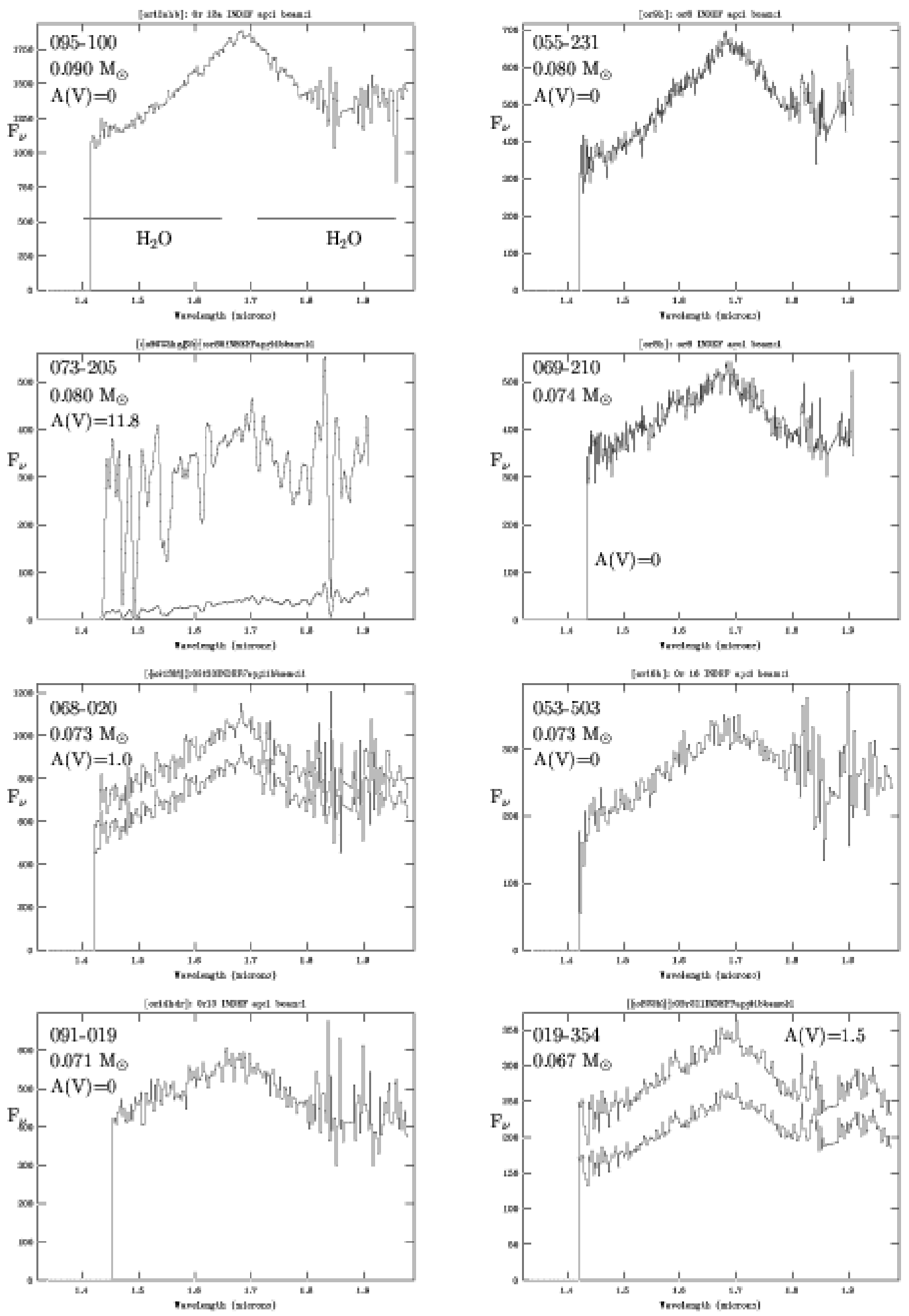

Figure 4(a): Observed $H$ bandpass spectra of Trapezium sources, with dereddened spectra overplotted as the upper line for each source when extinction is non-zero. Masses derived from the BM97 $1 \mathrm{Myr}$ isochrone are indicated. The noise is indicated by the pixel to pixel variations, since no narrow spectral features are detected in the $\mathrm{H}$ bandpass. 

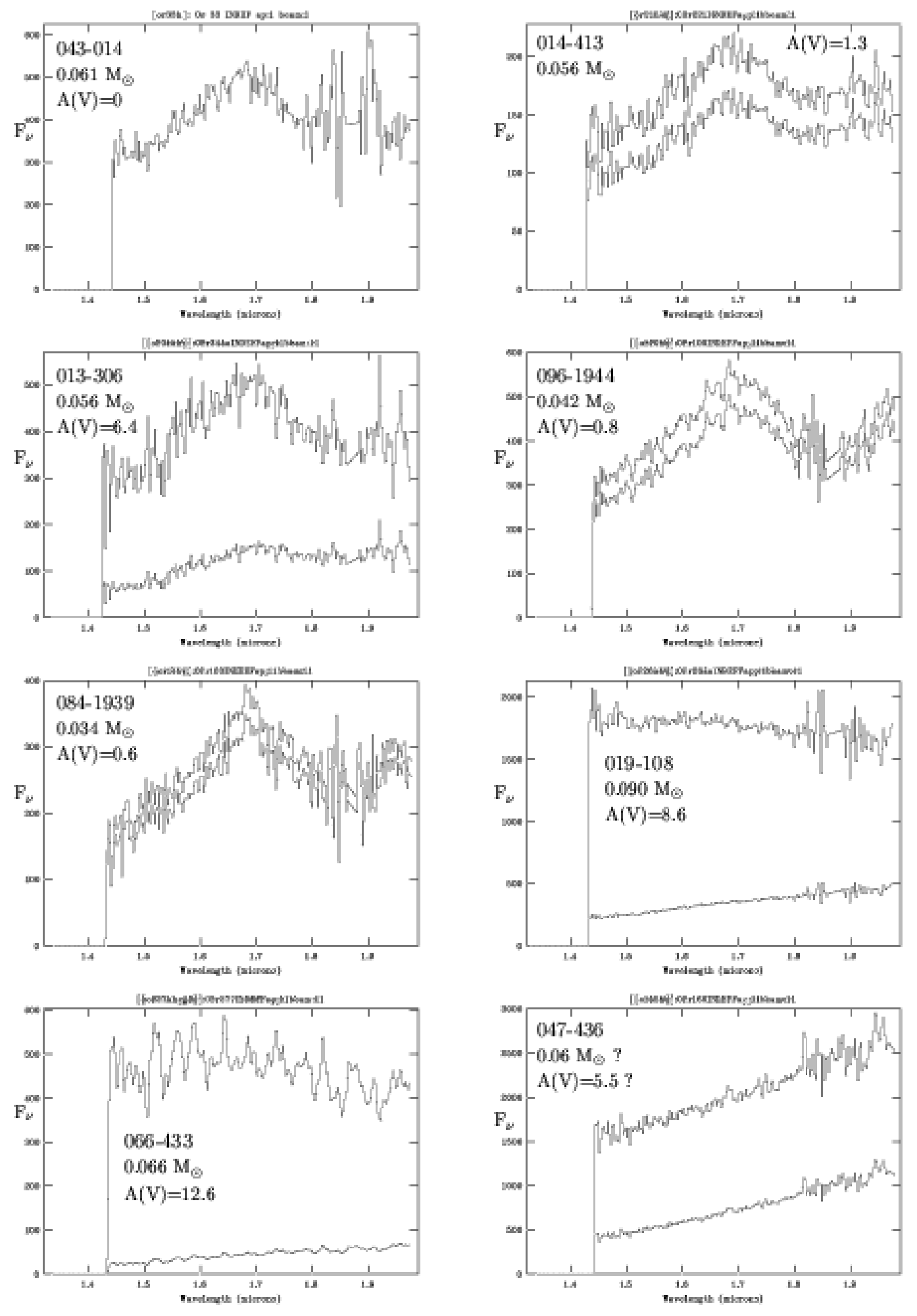

Figure 4(b): Observed and Dereddened $H$ bandpass spectra, as Figure 4(a). 047-436 shows no water absorption and 2 others have very weak water absorption. 

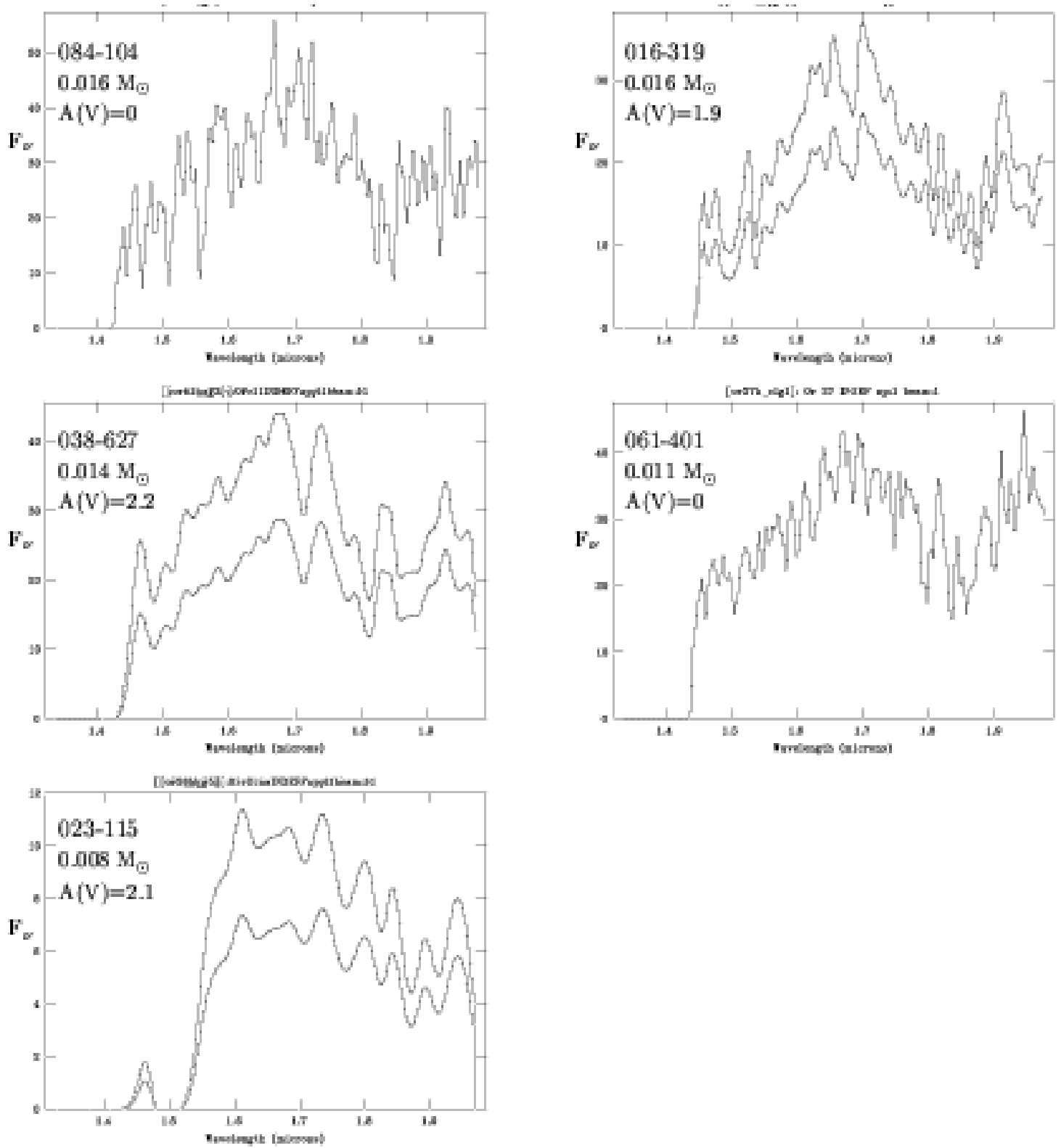

Figure 4(c): Observed and Dereddened $H$ bandpass spectra for the least massive candidates, with some gaussian smoothing applied. The apparent absence of flux at $\lambda \lesssim 1.5 \mu \mathrm{m}$ in 023-115 is attributing to the decline in signal/noise at those wavelengths, due to atmosphere, instrument efficiency, extinction and (presumed) water absorption in the source itself. 
The peaked $H$ bandpass profiles in Figure 4(a-c) are very different from those of late $\mathrm{M}$ and L-type dwarfs in the Local Neighborhood with strong water absorption bands, as shown by our spectra of 2 red standards in Figure 5(a-b), compared with a composite spectrum of the Orion sources. The composite spectrum is an average of 12 reasonably high quality spectra with fairly strong water absorption, weighted according to the apparent noise in each spectrum. The composite has slightly weaker water absorption than the average of the sample, since it is weighted toward warmer, brighter sources near the stellar/substellar boundary. Comparison with the data of Leggett et al.(2001) (overplotted in Figure 5(a)) for the same red standards demonstrates that the spectra are reliable and the differences from field stars are real. In spectra of field dwarfs there is strong water absorption at $\lambda<1.55 \mu \mathrm{m}$ and $\lambda>1.70 \mu \mathrm{m}$, but a shallower slope in between, which appears as a flat plateau in $\mathrm{F}_{\lambda}$ plots of the data (Figure 5(b)). This is clearly seen in the $\mathrm{F}_{\lambda}$ plots of a large sample of late $\mathrm{M}$ and $\mathrm{L}$ dwarfs presented by Leggett et al.2001. marked by clear changes in slope at $1.70 \mu \mathrm{m}$ and in the 1.55-1.60 $\mu \mathrm{m}$ interval. The peaked profiles of the Trapezium objects, which show no plateau, appear to be a consistent signature of youth and cluster membership. They are modelled in Section 4. There are no other clearly seen atomic or molecular features in the Trapezium sources at $H$ band, which is unsurprising given the low spectral resolution and modest signal to noise. Atomic absorption lines of $\mathrm{Na}$ and $\mathrm{Ca}$ have been observed in field dwarfs, eg. Jones et al.(1994) in high signal to noise spectra but many atomic lines appear to be weaker in very young brown dwarfs (eg. Bejar, Zapatero-Osorio \& Rebolo 1999.) The water absorption depths are quantified in Table 2, and discussed in Section 4.

Only $3 / 21$ sources have weak or zero water absorption. 019-108 and 066-433 suffer very high extinction $(\mathrm{A}(\mathrm{V})=$ 8.6, 12.6 respectively) and have very weak water absorption, consistent with late K-type or very early M-type spectra. These earlier spectral types are not consistent with the temperatures expected at age $1 \mathrm{Myr}$ even for these relatively luminous sources. (At $1 \mathrm{Myr}$ masses, $\mathrm{M}=0.090 \mathrm{M}_{\odot}$, $0.066 \mathrm{M}_{\odot}$ and $\mathrm{T}_{\text {eff }}=3400 \mathrm{~K}, 2800 \mathrm{~K}$ respectively are derived photometrically, using the BM97 isochrone). Therefore the $H$ band spectra indicate that they are either even younger and hotter systems (age $\sim 0.1 \mathrm{Myr}$ ) or they are background field stars. The dereddened fluxes of these stars, are faint enough to be consistent with late K-type dwarfs at $\mathrm{d} \geq 440 \mathrm{pc}\left(\mathrm{m}_{J}=13.72,14.45\right.$ for $019-108$ and $066-408$ respectively) judging by comparison with both the Luminosity Class V sequence of Tokunaga (2000) and the models of BCAH for low mass stars at late $\mathrm{K}$ dwarf temperatures. 019-108 would have to lie just behind the OMC-1 cloud at 440 pc, while 066-433 would lie at only a slightly greater distance. It therefore seems slightly more probable that these 2 hotter, highly reddened sources are background stars but the alternative of extreme youth is not excluded, especially since the stellar mass proplyd source 178-232 shows a similar spectrum (see below).

Only one source, 047-436, shows no water absorption in the $H$ bandpass, simply a very red continuum in Figure 4(b). This source is one of the $14 \%$ from Paper I with an anomalously blue $(I-J)$ colour (though not an extreme case, $\mathrm{I}=18.277,(I-J)=3.21)$ and so its extinction cannot be well measured. At present, we attribute such colours to light scattered by circumstellar matter. An attempt to apply the standard procedure leads to an insufficiently dereddened spectrum, shown in Figure 4(b). The youth and cluster membership of 047-436 is demonstrated by the $K$ bandpass spectrum, discussed below.

2 additional $H$ bandpass spectra of more massive sources are presented in Figure 6. 1 of these, 017-410 is a YSO with a mass of $\sim 0.3 \mathrm{M}_{\odot}$ which appeared in the slit by chance, owing to the crowded nature of the cluster. It shows fairly strong water absorption but more on the long wavelength side of the $1.7 \mu \mathrm{m}$ than the short wavelength side, which is different from the less massive sources, which may reflect the higher gravity of this source. Luhman et al.(2000) have obtained $K$ bandpass and optical spectra of stellar mass Trapezium sources but there do not appear to be many $H$ bandpass spectra of Trapezium stars in the literature to use for comparison. The second source is 178-232, a more massive star $\left(\mathrm{M} \sim 0.4 M_{\odot}\right)$ with an anomalously blue $(I-J)$ colour around which an emission line 'proplyd' structure was resolved by the Hubble Space Telescope (O'Dell \& Wong 1996). This has a very red spectrum, perhaps due to extinction by circumstellar matter rather than dust in the cluster medium and shows relatively weak water absorption, which would be expected for a hotter, younger object.

\subsection{2 $K$ band}

$11 K$ band spectra are shown in Figure $7(\mathrm{a}-\mathrm{b})$, and dereddened $H+K$ spectra are plotted in Figure $8(\mathrm{a}-\mathrm{b})$. The $H$ and $K$ spectra are joined by averaging the overlap region from 1.89-1.97 $\mu \mathrm{m}$, with some uncertainty $(\sim 10 \%)$ in the flux level owing to the telluric water absorption at those wavelengths. $9 / 11$ of the $K$ band spectra show strong water absorption bands on either side of a maximum near $2.25 \mu \mathrm{m}$, as expected for young brown dwarfs. The other 2 spectra, of 019-108 and 047-436, are consistent with very weak or zero water absorption, similar to the $H$ band data. The noise is indicated by the pixel to pixel variations as in the $H$ bandpass data.

The $\mathrm{v}=2-0$ vibration-rotation bands of $\mathrm{CO}$ at $\lambda>$ $2.29 \mu \mathrm{m}$ are detected in $4 / 11$ sources (see Table 3 ) via the edge at $2.29 \mu \mathrm{m}$ and marginally detected in one other source. Only one source, 053-503, shows the multiple bandheads clearly at $2.29,2.32,2.35$ and $2.38 \mu \mathrm{m}$ but they are also visible in 014-413. The weakness or absence of CO absorption is surprising for the low temperature photospheres indicated by the strong water absorption. However, it appears to be a feature of the youth and low gravity of cluster members, as demonstrated by the AMES-Dusty-1999 models in Section 4. Veiling by hot circumstellar dust cannot explain it, since the spectra decline steeply toward $2.5 \mu \mathrm{m}$, showing that water absorption is not significantly veiled at these wavelengths.

At $K$ band we might expect that hot dust would redden the spectra but if this is occurring the effect is too small to be unambiguously identified, except in the cases of 014-413 and 047-436, the only sources in Figure 8(a-b) showing much more flux in the $K$ bandpass than at $H$. 014-413 appears to be associated with a weak wind collison shock front in the data of Bally, O'Dell \& McCaughrean (2000) but shows no evidence of emission lines in the spectrum shown in Figure 

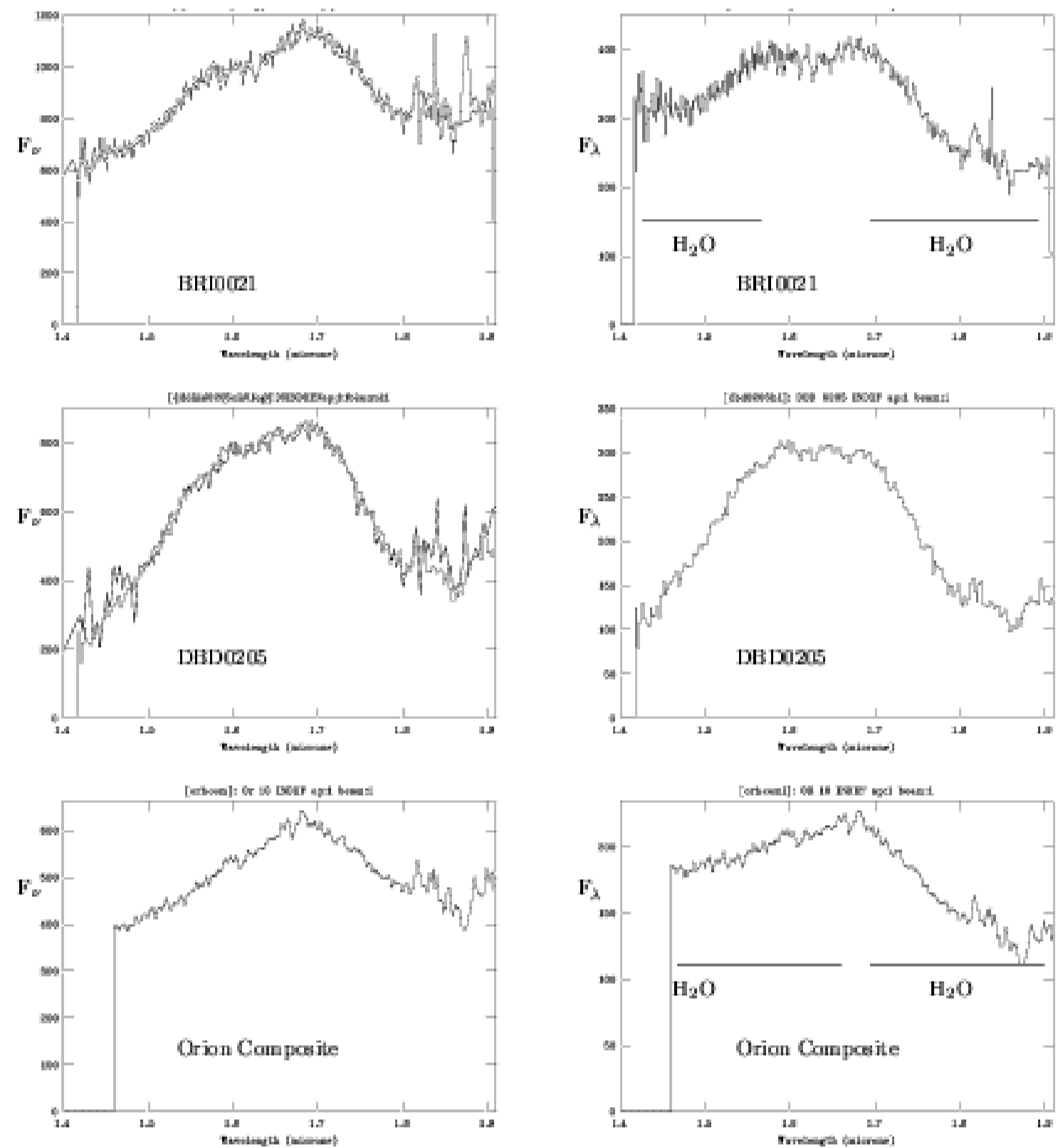

Figure 5(a-b): Red Standards and Orion composite spectrum. (a)(left) $\mathrm{F}_{\nu}$ spectra. Leggett et al.(2001) data are overplotted as a solid line for the field dwarfs to demonstrate the reliability of the spectra. (b)(right) spectra plotted as $\mathrm{F}_{\lambda}$, illustrating the plateau seen in field dwarfs in the $H$ bandpass. The composite is a weighted average of the warmer, more massive sources, so it has slightly weaker water absorption than the average of the sample. Weak narrow absorption features in the composite are generally attributed to telluric $\mathrm{OH}$ lines.

8(a). The possible effect of hot dust is shown in Figure 11, see Section 4. We do not have $K$ band photometric data but Hillenbrand \& Carpenter (hereafter HC) published $H K$ photometry of the central region of the Trapezium Cluster. In combination with extinction values derived from our $I J H$ data, these data should identify any sources with large $K$ band excesses. The HC data contains photometry for only 2 of the 11 sources in our $K$ band spectroscopic sample (which lies to the west of the cluster core). Their $H$ band fluxes of $\mathrm{HC}$ agree with our data to within 0.18 magnitudes for the 2 sources, which is broadly consistent with the photometric uncertainties but may indicate T Tauri-like variability. Nei- ther of these has any $K$ band photometric excess, which is consistent with the spectra. Some sources in the much larger photometric dataset do appear to have $\mathrm{K}$ bandpass excesses (see Section 6 and Table 4). Wilking, Greene \& Meyer calculated the contribution of hot dust using $J H K$ photometry for low mass stars and brown dwarfs in the $\rho$ Ophiuchus star forming region. They found that hot dust contributed $0 \%$ to $20 \%$ of the total flux in the $K$ bandpass in the majority of cases, which would have only a small effect on spectral profile. Star formation is nearly complete in the Trapezium Cluster and the average age of this sample of low-extinction sources is probably greater than that of sources in $\rho$ Ophi- 

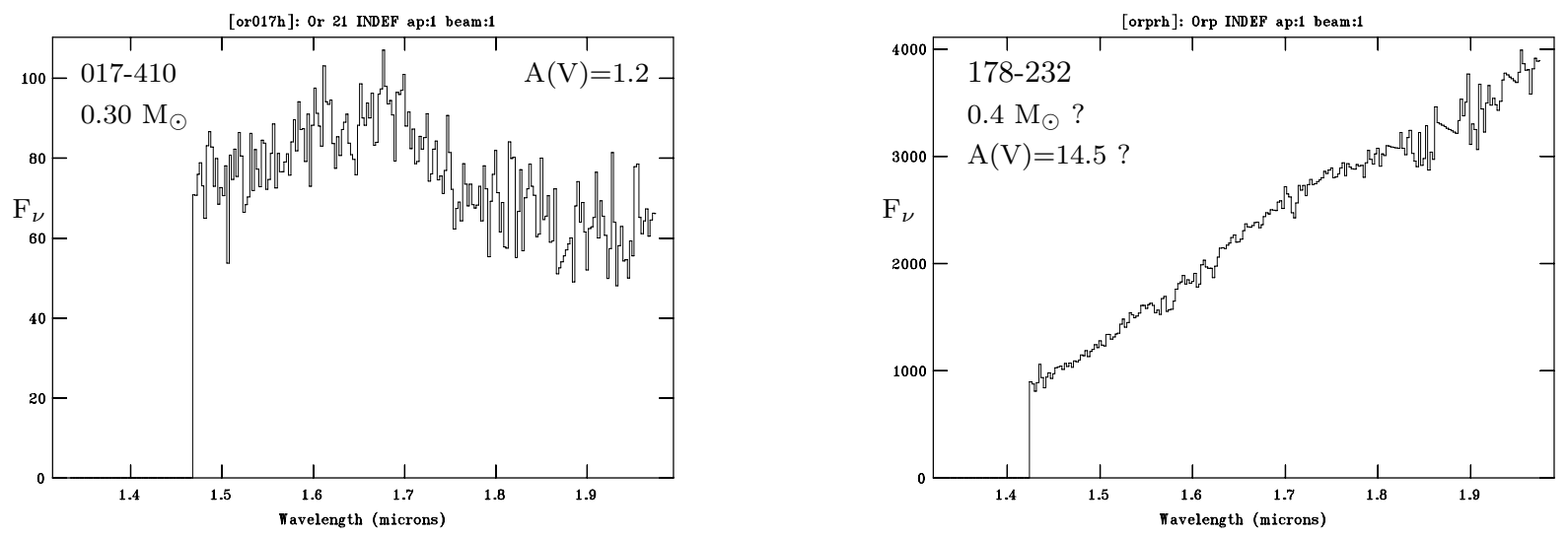

Figure 6. Observed $H$ bandpass spectra of 2 more massive sources (0.3-0.4 $\left.\mathrm{M}_{\odot}\right)$. 178-232 is a proplyd; the weak water absorption and red spectrum is consistent with an embedded, warmer source.

uchus so it is not too surprising that we do not see many large infrared excesses here.

The NaI line at $2.21 \mu \mathrm{m}$ is the strongest atomic aborption line in the $H$ and $K$ band spectra of field dwarfs (see Jones et al. 1994). It is clearly visible in the $K$ band spectra (see Figure 16) but is not seen in the Trapezium sources. We would expect it to be seen in the high quality spectrum of 053-503 (see Figure 16) but there is only a small local minimum near that wavelength, which cannot be distinguished from noise. This weakness of this feature is also predicted by the AMES-Dusty-1999 models as a signature of low gravity, as described in Section 4.1.2.

The $K$ band spectrum of 047-436 (Figure $7(\mathrm{~b})$ ) has a very red continuum in which water absorption is weak or absent, following the trend seen at $H$ band. However, there is a rich sequence of $\mathrm{H}_{2}$ emission lines, with strong lines at $2.12,2.41$ and $1.96 \mu \mathrm{m}$ and several weaker lines apparent also (see Table 2). As mentioned above, this is an anomalously blue source in the $I$ band and these are usually also resolved as proplyds by the Hubble Space Telescope (O'Dell \& Wong 1996), as discussed in Paper I. This particular source is listed as being stellar in the data of O'Dell and Wong however, perhaps because it is located a large distance (3 arcmin, or $0.4 \mathrm{pc}$ ) from the cluster centre, leading to less excitation of the optical emission lines that mark a proplyd, and insufficient contrast with the faint nebulous background for a silhouette disk to be observed. 047-436 lies $<1$ arcmin north of the complex of bow shocks associated with LL Ori., observed in $\mathrm{H} \alpha$ by Bally et al.(2000).

The $\mathrm{H}_{2}$ emission is the signature of a very young system with plentiful circumstellar gas. Such a signature can be caused by shocked gas in a molecular outflow or by UV excitation of gas in a circumstellar accretion disk or envelope i.e. a proplyd similar to those discovered by O'Dell \& Wen (1994). In Orion the UV radiation is supplied mostly by $\theta_{1}$ Ori C. Chen et al.(1998) resolved the $2.12 \mu \mathrm{m} \mathrm{H} \mathrm{H}_{2}$ from 2 proplyds with NICMOS and found that it was confined to the subarcsecond disk silhouettes seen in continuum imaging. However, the emission from the 3 strongest $\mathrm{H}_{2}$ lines in 047-436 is extended along the slit of the spectrograph by at least $3 \operatorname{arcsec}(1300 \mathrm{au})$ in a southerly direction, the peak of the emission being coincident with the continuum source.
There is no extension in the continuum and seeing conditions were fairly good $(\sim 0.6$ arcsec $)$ at the time the spectrum was taken. The slit was oriented $18^{\circ}$ west of north. The large extension of the $\mathrm{H}_{2}$ emission in $047-436$, relative to the Chen et al. proplyds, may be due to the high extinction and much larger projected distance from the Trapezium stars, which would allow molecular material to survive to survive for a greater period of time. The reduced group image is not very sensitive to larger scale emission because a nod length of only 10 arcsec was used in taking the spectra and subtracting the background. However, the extended emission appears to be weaker in the northerly direction, extending for $<1.5$ arcsec. The strength of the emission in the southern direction declines monotonically with distance from the peak, dropping by a factor of $\sim 2$ at a distance of 2 arcsec from the central source. There do not appear to be any $\mathrm{H}_{2}$ emission line spectra of proplyds in the literature, although there are spectra of shocked gas in the vicinity of the BN object. Theoretical models of optical proplyd spectra, eg. Storzer \& Hollenback (1999) predict that some of the lines, eg. the (1-0) $\mathrm{S}(1)$ line at $2.12 \mu \mathrm{m}$, may be collisionally excited in the dense environment of the circumstellar disk, not excited by UV fluorescence. This makes it more difficult to understand the relative strengths of the emission lines and a full analysis is beyond the scope of this paper. However, the ratio of the (2-1) $\mathrm{S}(1)$ and (1-0) $\mathrm{S}(1)$ line strengths in Table 2 does suggest UV fluorescence.

We conclude that 047-436, the only source entirely lacking $H$ bandpass water absorption, is definitely a member of the Trapezium cluster. The slope of the 'dereddened' spectrum in Figure $8(\mathrm{~b})$ is not very reliable because it is an anomalously blue source $(I-J)$ source. It is quite possible that hot dust contributes to the $K$ band spectrum in this very young system but the bulk of the very strong reddening in the $H$ bandpass must be due to extinction, perhaps by circumstellar dust. A very young system (age of order $0.1 \mathrm{Myr}$ ) would have a hotter photosphere than the rest of the sample, so any water absorption would be weak and in this case veiled by extinction. The mass of 047-436 can be estimated as $0.06 \mathrm{M}_{\odot}$ from $I J H$ photometry in Paper I, assuming an extinction of $\mathrm{A}(\mathrm{V})=5.5$. A $0.1 \mathrm{Myr}$ source would be less massive than a $1 \mathrm{Myr}$ source for a given luminosity 

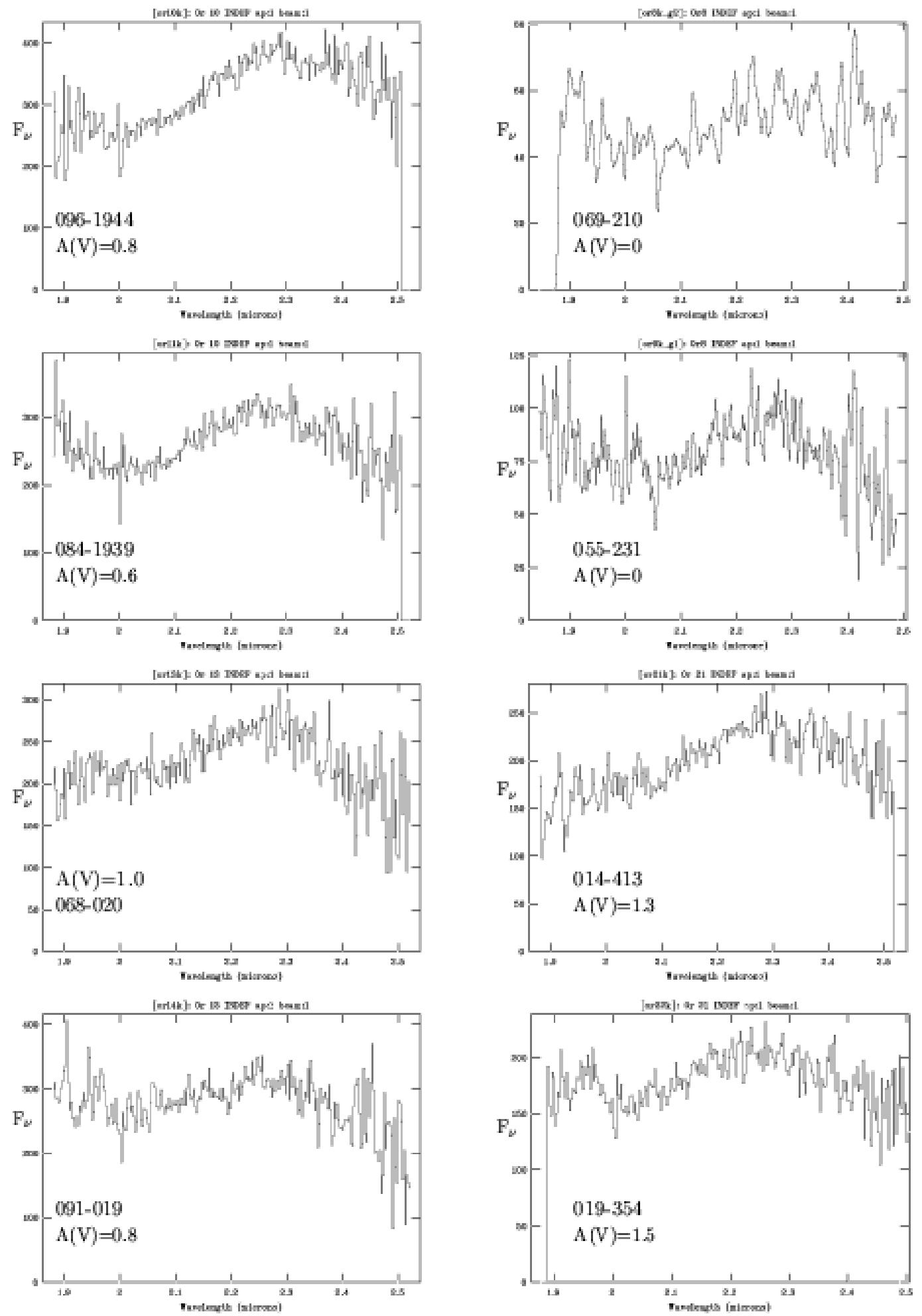

Figure 7(a): Observed $K$ bandpass spectra of Trapezium sources. The noise is indicated by pixel to pixel variations, as in Figure 4(a-c). 069-210 and 055-231 are gaussian smoothed, because they were observed in poor conditions. 

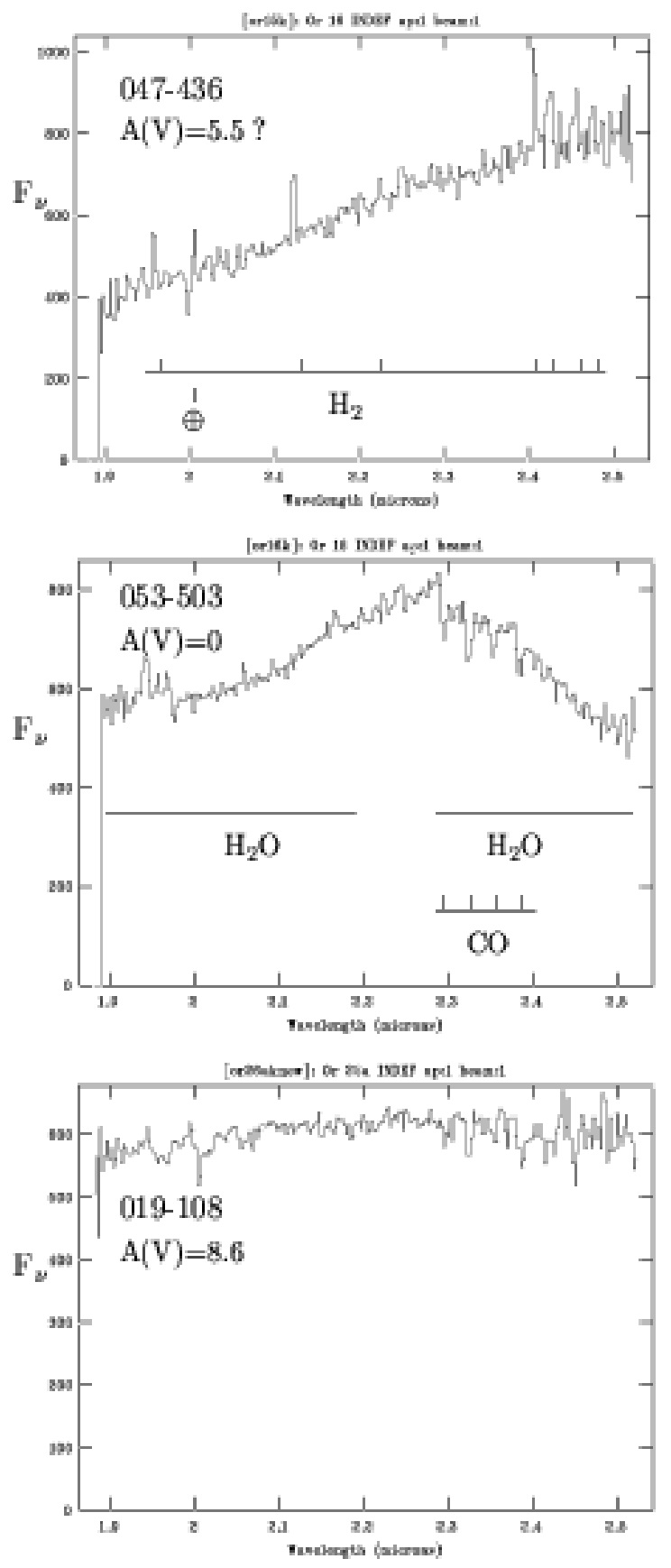

Figure 7(b): Observed $K$ bandpass spectra of Trapezium sources. The stronger $H_{2}$ emission lines in 047-436 are marked, together with a telluric feature at $2.0 \mu \mathrm{m}$.

but an extinction of $\mathrm{A}(\mathrm{V})=10$ is required to produce a flat $\mathrm{F}_{\nu}$ spectrum, which might be more appropriate. Hence, the mass is highly uncertain and it is possible that it is a very low mass star rather than a brown dwarf.

Object 019-108 also has high extinction and shows only weak water absorption at $H$ but its $K$ bandpass spectrum shows no sign of emission by $\mathrm{H}_{2}$ or by hot dust. This suggests that it is a background star rather than a cluster member, as discussed in Section 3.1. However the evidence is not conclusive: circumstellar matter could be entirely swept away by the photoionising UV radiation field in $\ll 1$ Myr (eg.
Storzer \& Hollenback), so it might be a very young cluster member.

\section{ANALYSIS}

We analyse the spectra by comparison with both the AMES-Dusty-1999 model spectra calculated by Allard et al. $(2000,2001)$ and with local field dwarfs. Since the spectra are dominated by water absorption bands, a water index of some sort is the natural tool to use to derive spectroscopic effective temperatures $\left(\mathrm{T}_{e f f}\right)$. We do not fit the full 1.4$2.5 \mu \mathrm{m}$ spectra to those of field dwarfs or giants, after the fashion of Luhman et al.(1998a) at optical wavelengths since the $H$ bandpass profiles are clearly different (see Section 1). The synthetic spectra are believed to be most accurate at $\lambda \leq 1.7 \mu \mathrm{m}$ (see Section 4.1). In addition, the most strongly temperature sensitive broad band feature at the observed wavelengths is the wing of the $1.35 \mu \mathrm{m}$ water band in the 1.4-1.7 $\mu \mathrm{m}$ region, the other bands being nearly saturated for all late $\mathrm{M}$ and $\mathrm{L}$ spectral types. Hence we define a water index $W=\mathrm{F}_{\nu}(1.50 \mu \mathrm{m}) / \mathrm{F}_{\nu}(1.682 \mu \mathrm{m})$. The model spectra are rebinned to the same dispersion as the data and convolved with the instrumental profile as measured in the Arc spectra. For the model spectra the 1.5 and $1.682 \mu \mathrm{m}$ fluxes are averaged over $0.02 \mu \mathrm{m}$ (or 9 data points) at both wavelengths, while the observed spectra have their 'continuum' fitted with high order cubic splines to reduce the measurement errors. The 1.50-1.682 $\mu \mathrm{m}$ range covers most of the depth of this water absorption band, the data being less precise at shorter wavelengths. The model spectra peak at $1.682 \mu \mathrm{m}$ in the $2500-3300 \mathrm{~K}$ range and the dereddened data also peak at or very close to this wavelength.

The choice of water index is always somewhat arbitrary, so we also compute a reddening independent index of the type used by Wilking, Greene \& Meyer at $K$ band, but applied here to the $H$ band spectra. This index is defined by $Q=(F 1 / F 2)(F 3 / F 2)^{1.219}$, where $\mathrm{F} 1$ is the $\mathrm{F}_{\nu}$ flux at $1.57 \mu \mathrm{m}, \mathrm{F} 2$ is the $\mathrm{F}_{\nu}$ flux at $1.682 \mu \mathrm{m}$ and $\mathrm{F} 3$ is the $\mathrm{F}_{\nu}$ flux at $1.79 \mu \mathrm{m}$. The 1.57 and $1.79 \mu \mathrm{m}$ wavelengths are chosen because they must be roughly equidistant from the flux peak for good precision and because the data is often of poor quality at $\lambda \gtrsim 1.8 \mu \mathrm{m}$, and therefore not used in the continuum fitting process. We also tested an index similar to that of Delfosse et al.(1999), based on the slope in the 1.51-1.57 $\mu \mathrm{m}$ region. However, the measurement errors for the slope in this narrow region are generally too large for that index to be very useful here and $K$ bandpass data is required to normalise the index as defined by Delfosse et al. The measured $W$ and $Q$ indices are listed in Table 3, along with spectroscopic temperatures derived by fitting the $W$ indices to those of the models. Temperatures are rounded to the nearest $50 \mathrm{~K}$, since the model grid has an interval of $100 \mathrm{~K}$.

We assign approximate spectral types to each source (see Table 3), by the comparison of their $W$ and $Q$ indices with those of the local field dwarfs observed by Leggett et al.(2001), whose spectra types are defined using the 2MASS Kirkpatrick et al.(1999) system. The reddening independent $Q$ index should be the more reliable for measuring water depth and for spectral typing but the $W$ index generally produces very similar spectral types, as shown in the Table. 

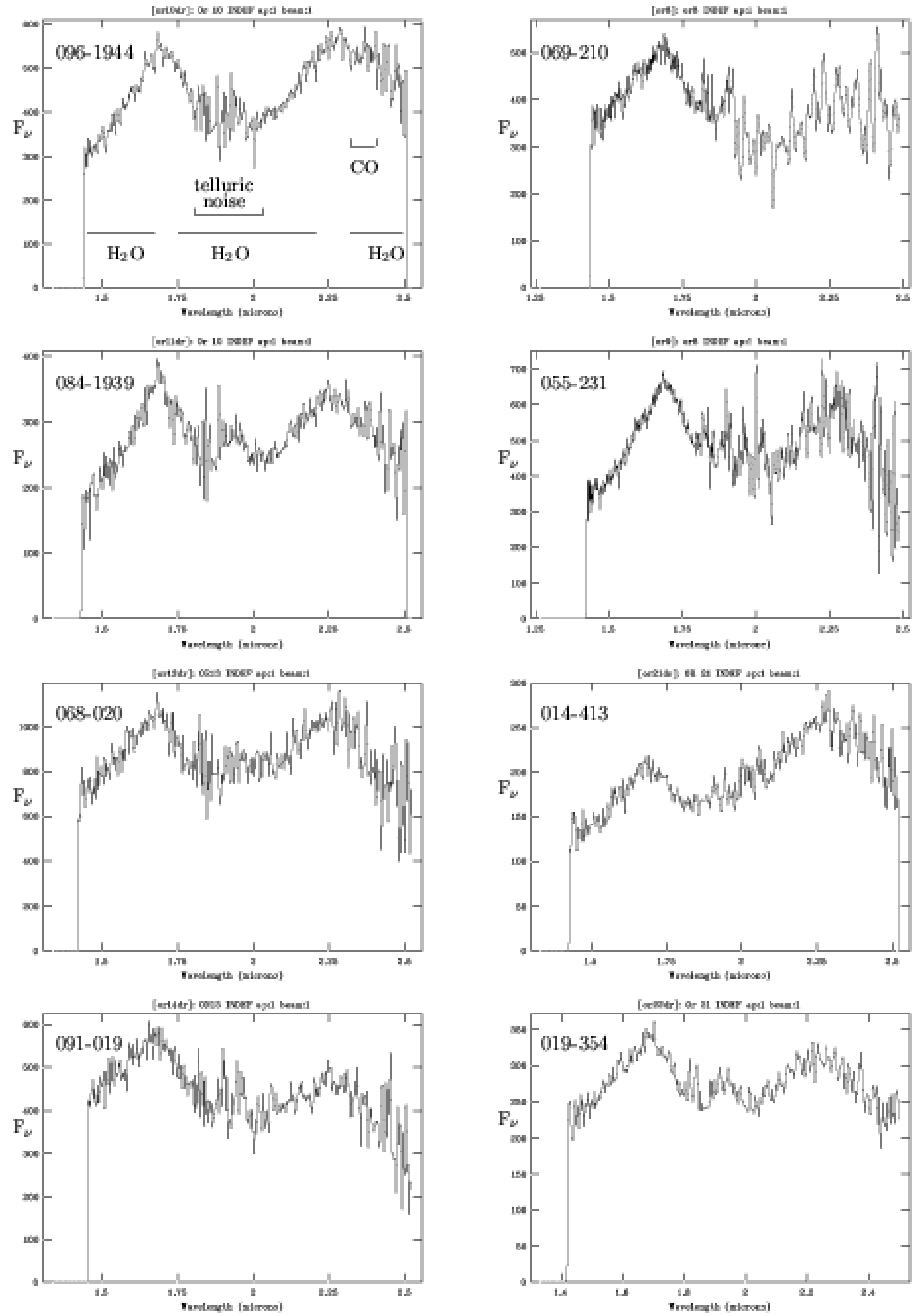

Figure 8(a): Dereddened spectra for Trapezium sources observed at both $H$ and $K$. Noise in the $1.8-2 \mu \mathrm{m}$ region is due to time variable telluric water absorption. 

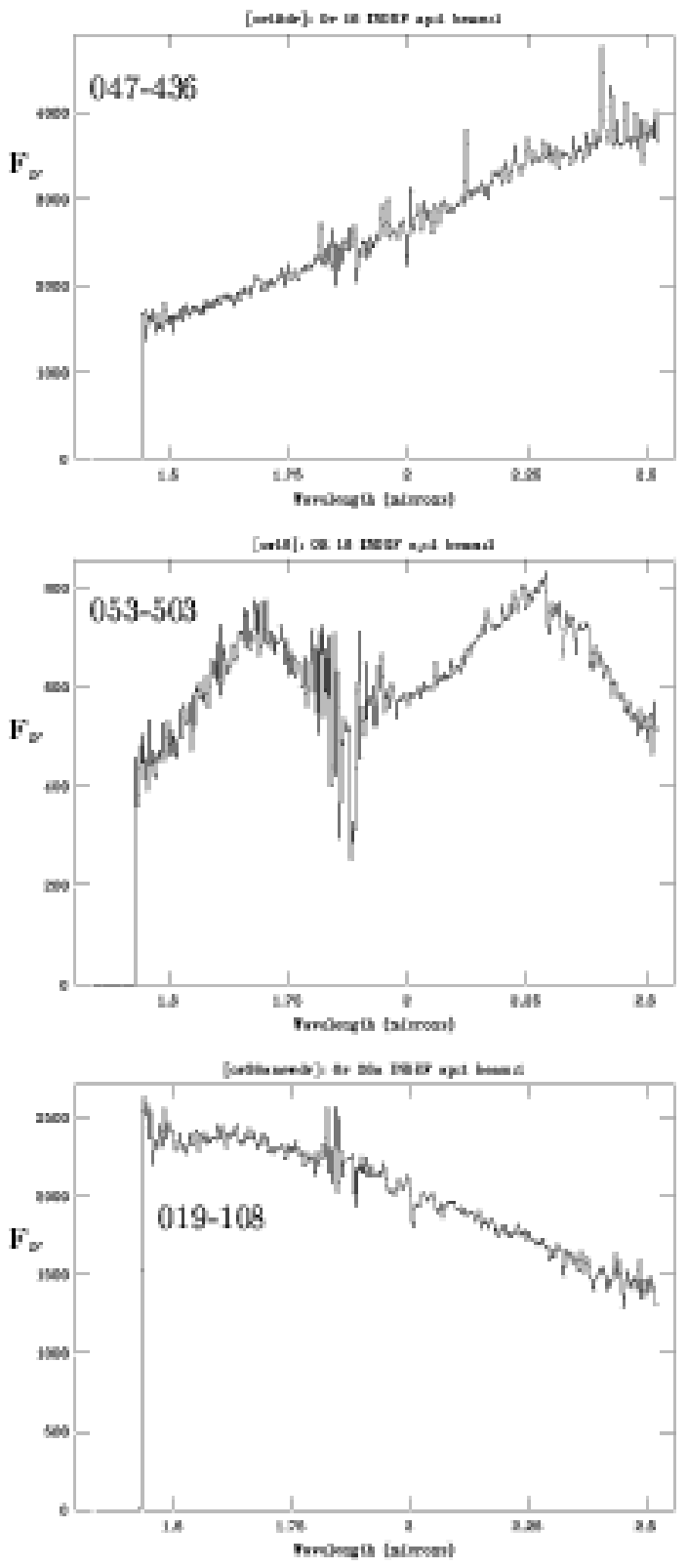

Figure 8(b): Dereddened spectra for Trapezium sources observed at both $H$ and $K$.

It is important to note that these are not real spectral types as might be defined at optical wavelengths - they are merely the spectral types that the $H$ bandpass water depth would correspond to if the the Trapezium sources were high gravity field dwarfs. Low gravity sources have stronger water absorption for a given $\mathrm{T}_{\text {eff }}$ than field dwarfs. Hence the spectral types appear rather late for the corresponding spectroscopic temperatures in Table 3 and we would expect many of the L-type sources to have late M-type spectra at optical wavelengths. The behaviour of the $W$ and $Q$ indices as a function of spectral type is shown in Figure 9 for the field dwarfs. Most of the 15 field sources follow a linear relation quite well but there is some interesting scatter (which is definitely real). Gd165b is an L4 dwarf (in fact the prototypical object) but has very weak water absortion for its spectral type. The 2 M9 dwarfs in the Leggett al.(2001) sample show large scatter on either side of the best fit lines, particularly in the $W$ index. Some of these differences might be attributed to dust effects: dust in cool metal rich atmospheres is expected to heat the photosphere; this may be influenced by metallicity, rotation and age, as noted by Leggett et al. However, there is no clear difference between the water depths of Young Disk and Old Disk sources in the sample, categorised kinematically by Leggett et al.(2001). Another study by Leggett et al.(2000) showed little difference due to metallicity in the $H$ bandpass when comparing Disk dwarfs and Halo subdwarfs but much weaker water absorption in Halo extreme subdwarfs, in which the $H$ bandpass profile is dominated by $\mathrm{H}_{2}$ opacity in early M-type dwarfs.

The spectral types of the Trapezium sources lie in the range M7-L5 for 16 of the 18 low mass sources $\left(<0.1 \mathrm{M}_{\odot}\right)$ which show strong water absorption. The 2 objects with later spectral types are faint and might have imperfect background subtraction (see Section 2). The fitted spectroscopic temperatures $(2100,2350 \mathrm{~K})$ are consistent with the new predictions for planetary mass sources by Baraffe et al. and by BM97 but are slightly low for these 2 sources with $\mathrm{M} \sim 0.016 \mathrm{M}_{\odot}$ at $1 \mathrm{Myr}$, unless they are younger, less massive objects of equivalent luminosity but greater size. It might be useful to define a new luminosity class or spectral type to describe these young, low gravity objects but since the gravities increase continuously over a period of several hundred Myr this is probably not a valuable concept. Instead we regard these low mass sources as a low luminosity extension to the region occupied by $\mathrm{T}$ Tauri stars on the HR diagram.

\subsection{Synthetic Spectra}

The AMES-Dusty-1999 spectra appear to represent a significant improvement over previous models (such as NextGen) of cool dwarfs at infrared wavelengths, as discussed by Allard, Hauschildt \& Schwenke (2000, hereafter AHS). Models using the Miller-Tennyson list do not accurately reproduce the infrared profiles of either these data or field dwarfs (see Fig.9 of Leggett et al.2001). By contrast the AMES-Dusty1999 spectra, which use the newer NASA Ames water line list, provide quite a good fit to the $H$ bandpass profiles of the Orion data (see Figure 10(a-d)) and go some way toward reproducing to the plateau seen in spectra of field dwarfs. The synthetic spectra decline a little more steeply on the long side of the $H$ bandpass than the data, unless $\mathrm{T}_{\text {eff }} \gtrsim 3300 K$. Such a high temperature would not be consistent either with the photometric temperatures obtained from the dereddened $(I-J)$ colours in Paper I, or the evolutionary predictions of BM97 and Baraffe et al. As noted in Section 3.1, there appears to be incompleteness in the water line list for the absorption band centred near $1.95 \mu \mathrm{m}$. This compromises the predicted spectral profiles in the 1.7 to $2.2 \mu \mathrm{m}$ region and leads to small errors in the predicted $H$ and $K$ bandpass fluxes, as discussed by AHS. By contrast the water absorption band centred at $1.35 \mu \mathrm{m}$ is well fitted by the AMES-Dusty-1999 spectra (see AHS) and the fluxes and spectral profiles are believed to be more accurate at $\lambda \leq 1.7 \mu \mathrm{m}$. It is generally not possible to fit both sides of the $H$ bandpass spectra simultaneously. This is one of the reasons why the short wavelength side is used to de- 

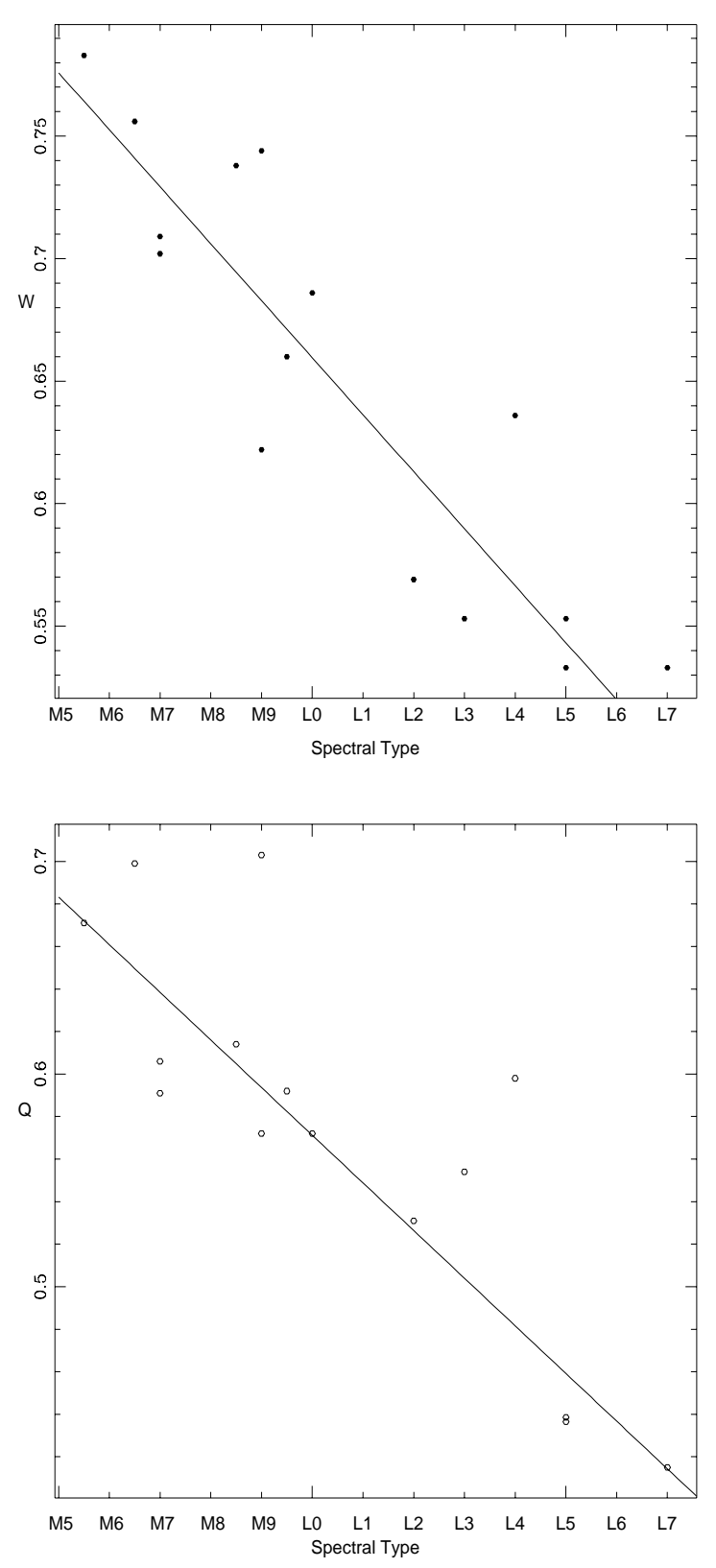

Figure 9: $W$ and $Q$ spectral indices as a function of spectral type for Field Dwarfs. Best fit lines are plotted, excluding the divergent L4 source and the 2 M9-types. Including these would lead to later types for the coolest sources. Measurement errors are very small for these nearby sources.

rive spectroscopic temperatures, the other being the greater temperature dependence of that part of the synthetic spectra.

The discrepancy at 1.7-1.9 $\mu \mathrm{m}$ seen in Figure 10(a-d) could in principle be caused by hot circumstellar dust emitting detectable flux on the long wavelength side of the $H$ bandpass. However, as shown in Figure 11, this would lead to strong and obvious excess emission in the $K$ bandpass spectra, and some veiling of the water absorption near $2.5 \mu \mathrm{m}$, which is not seen in most of the sources in Figure 8(a-b).

The evolutionary calculations of BM97, D'Antona \& Mazzitelli (1998) and Baraffe et al. predict surface gravi- ties between $\log (\mathrm{g})=3.5$ and $\log (\mathrm{g})=4.0$ for sources of brown dwarf and planetary mass in the 0.3-2 Myr age range expected for most Trapezium sources. AMES-Dusty-1999 spectra in this gravity range reproduce the constant slope of the data at $\lambda<1.68 \mu \mathrm{m}$ and the wavelength of the flux peak. Higher gravity synthetic spectra show a flatter profile near the flux peak, and a steeper slope at shorter wavelengths. This is most easily seen in an $F_{\lambda}$ plot, shown in Figure 12, which may be compared with the composite Orion spectrum and field dwarf spectra in Figure 5(b). This is qualitatively more similar to the profile of field dwarfs (with $\log (\mathrm{g}) \approx 5.5$ ), although the abrupt change in slope observed in the 1.55$1.60 \mu \mathrm{m}$ interval is not reproduced. The higher gravity models also decline more steeply on the long wavelength side of the $H$ bandpass than the low gravity ones, which leads to a greater discrepancy with the data at those wavelengths than is seen in the low gravity fits in Figure 10(a-d).

In the $K$ bandpass the behaviour of synthetic spectra is somewhat similar to that in the $H$ bandpass but less clear cut. The low gravity models have a fairly constant slope on the wing of the water absorption band in the $2.05-2.25 \mu \mathrm{m}$ region, whereas high gravity models show a curved profile similar those in the $H$ bandpass. The water vapour absorption is less deep at $K$ bandpass and the profile of the flux peak is apparently affected by $\mathrm{H}_{2}$ absorption longward of $2.2 \mu \mathrm{m}$ in some field L dwarfs (Tokunaga \& Kobayashi 1999). One might argue that some of the spectra shown in Figure 8(a-b), such as the high quality spectrum of 053-503, lack the flat profile seen in $K$ bandpass $\mathrm{F}_{\nu}$ spectra of late $\mathrm{M}$ and $\mathrm{L}$ dwarfs (eg. Jones et al.1994) but there is more variation between sources than at $H$ band, both in our data and in field dwarf spectra. Owing to these factors and the relatively poor quality of most of our $K$ band spectra the water absorption bands are not clearly distinguished from those of field dwarfs.

The spectroscopic temperatures fitted in Table 3 generally lie in the range $2150-3300 \mathrm{~K}$ for the $18 / 21$ sources in the sample which have strong water absorption. All but 2 sources have $T_{\text {eff }} \geq 2500 \mathrm{~K}$, so there is insufficient dust in the photospheres to have a significant effect on the synthetic spectra. The fitted values of $\mathrm{T}_{\text {eff }}$ differ by some $450 \mathrm{~K}$ between $\log (\mathrm{g})=3.5$ models and $\log (\mathrm{g})=4.0$ models at the high temperature end, with the higher gravity models giving lower temperatures. At $2500 \mathrm{~K}$ and below the same temperatures are fitted for both gravities. These temperatures are in good agreement with the evolutionary calculations of BM97. in the 0.008-0.080 $\mathrm{M}_{\odot}$ mass range, for which they predict $2150<T_{\text {eff }}<3230$ at $1 \mathrm{Myr}, 2310<T_{\text {eff }}<3290$ at $0.3 \mathrm{Myr}$ and $2030<T_{\text {eff }}<3180$ at $2 \mathrm{Myr}$. The Baraffe et al. and D'Antona \& Mazzitelli models generally predict very similar temperatures to BM97 (within 50-150 K) which in our view is less than the uncertainty in the calibration of the absolute temperature scale with observed spectral type. The $\log (\mathrm{g})=4.0$ models produce better agreement for the higher mass sources of the sample than the $\log (\mathrm{g})=3.5$ models.

The spectroscopic temperatures fitted at $\log (\mathrm{g})=4.0$ also agree well with the photometric temperatures derived from the dereddened $(I-J)$ colours from Paper I, by comparison with the colours of field dwarfs (predicted for main sequence stars by BCAH using the NextGen model atmospheres). This is illustrated in Figure 13, which shows an excellent correlation between the two independently derived 

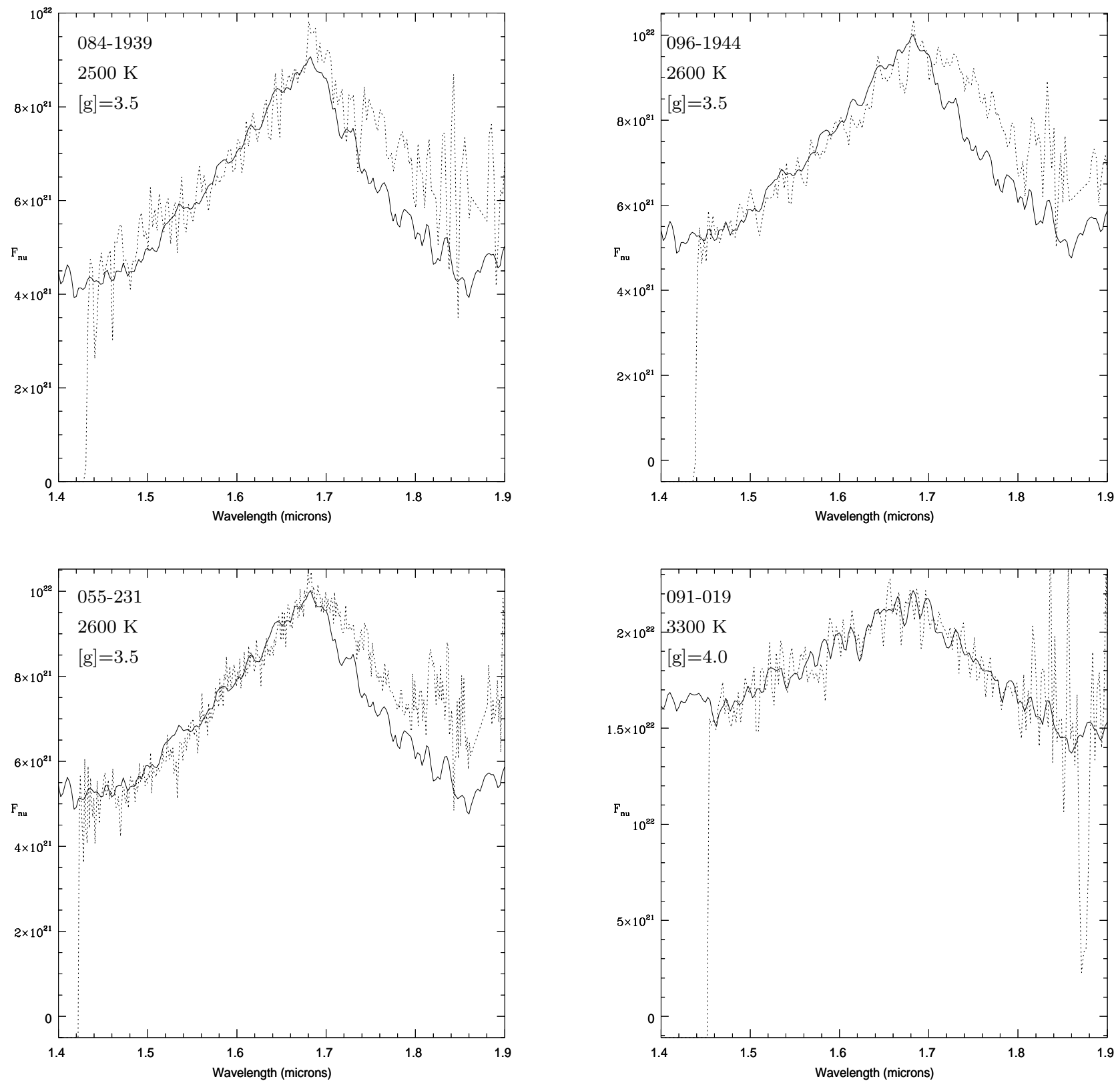

Figure 10(a-d): AMES-Dusty-1999 temperature fits (solid lines) to 4 sources (dotted lines), fitted via the $W$ index. The 1.4-1.7 $\mu \mathrm{m}$ region and the peak are well fitted. The 1.7-1.9 $\mu \mathrm{m}$ region cannot generally be fitted simultaneously, except in the warmest sources (see text). The discrepancy increases for higher gravities.

temperature scales but a systematic offset toward lower temperatures of about $250 \mathrm{~K}$ in the photometric temperatures. The scatter is largely due to photometric measurement errors magnified by dereddening rather than the spectroscopic measurements, and fits in well with the estimated uncertainty of $0.25 \mathrm{mag}$ in the dereddened $(I-J)$ colours given in Paper I. The NextGen atmospheres are known to fit main sequence infrared colours quite well, (better then the AMESDusty models at $\mathrm{T}_{\text {eff }}>2500 \mathrm{~K}$ ) despite the very poor fit to the spectral profiles at $H$ and $K$ seen by Leggett et al.(2001). Hence the $250 \mathrm{~K}$ offset must be due either to the lower gravity of the sample or errors in the AMES-Dusty models. The
NextGen spectra predict that $(I-J)$ colours differ by only of order 0.1 mag between $\log (\mathrm{g})=3.5$ models and $\log (\mathrm{g})=5.5$ sources, which corresponds to an interval in temperature of $\sim 50 \mathrm{~K}$. However, the lower gravity also changes the predicted $(J-H)$ colours by $\sim 0.1 \mathrm{mag}$, which leads to a larger difference in the $(I-J)$ colours via the dereddening process. Hence, it is quite possible that the offset of $250 \mathrm{~K}$ is due solely to gravity effects, and the spectroscopic temperatures at $\log (\mathrm{g})=4.0$ are correct. They are more consistent with the evolutionary calculations for age $1 \mathrm{Myr}$ than the photometric temperatures and are derived from the well-fitted 1.4-1.7 $\mu \mathrm{m}$ region. Broad band $(J-H)$ fluxes produced by 


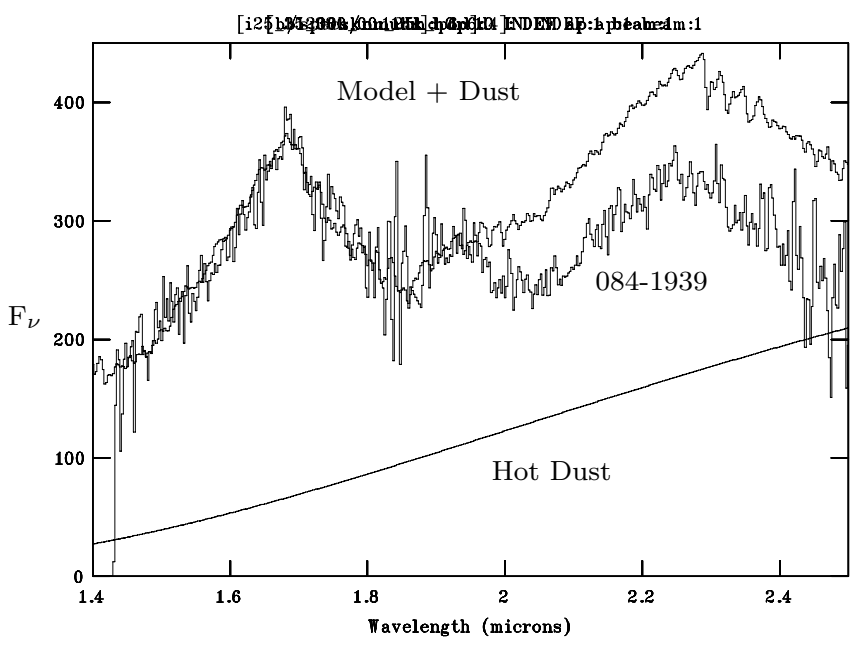

Figure 11: Hot dust. Strong emission by dust at $1200 \mathrm{~K}$, corresponding to excess $\mathrm{E}(\mathrm{H}-\mathrm{K})=0.5$ is added to an AMES-Dusty model. This can fill in the discrepancy at 1.7-1.9 $\mu \mathrm{m}$ but leads to a very obvious excess in the $K$ bandpass.

the NextGen and AMES-Dusty model atmospheres at these gravities are not yet more reliable than the assumption of main sequence colours, owing to the problems with the water line list referred to above. (The AMES-Dusty models produce most accurate colours for high gravity field dwarfs below $2500 \mathrm{~K}$, while the NextGen models work best at higher stellar temperatures, above $3000 \mathrm{~K}$ ). Hence, at present we continue to use empirical main sequence colours in the dereddening procedure.

There is a trend for less luminous, less massive sources to be cooler (see Figure 14), as indicated by the trend for water absorption to increase with decreasing mass. The BCAH models predict that water absorption increases monotonically with decreasing temperature in these substellar premain sequence objects. A mass-temperature relation correlation is predicted by all the published pre-main sequence isochrones. The derived luminosities, and hence masses, are nearly independent of temperature owing to the very weak temperature dependence of the $(J-H)$ colour so this is not a trivial result. The correlation is imperfect, presumably because of the dispersion in ages.

\subsubsection{Carbon Monoxide and other molecules}

The sythetic spectra also show a strong gravity dependence in the strength of the CO absorption bands near $2.3 \mu \mathrm{m}$, which is largest at low temperatures. This is illustrated in Figure 15, with $K$ bandpass spectra of high and low gravity model atmospheres at $2500 \mathrm{~K}$ and $2900 \mathrm{~K}$. We define a a CO index, $K_{C O}=(F 22-F 23) / F 22$ where F23 is the integrated $\mathrm{F}_{\lambda}$ flux in the $2.295-2.315 \mu \mathrm{m}$ region and $\mathrm{F} 22$ is the integrated $\mathrm{F}_{\lambda}$ flux in the $2.2675-2.2875 \mu \mathrm{m}$ region. The indices for models and data are listed in Table 3. All of the Trapezium sources have $K_{C O}<0.12$, i.e. an average absorption depth across the first CO band of $<12 \%$. The observed CO indices of 0.09-0.12 observed in 053-503, 014-413 and 055-231 are in good agreement with the model predictions for $\log (\mathrm{g})=3.5$ to 4.0 and $2500<T_{\text {eff }}<3200 \mathrm{~K}$. The data are much less consistent with high gravity models, particularly for the cooler sources. For example, we observe $K_{C O}=$
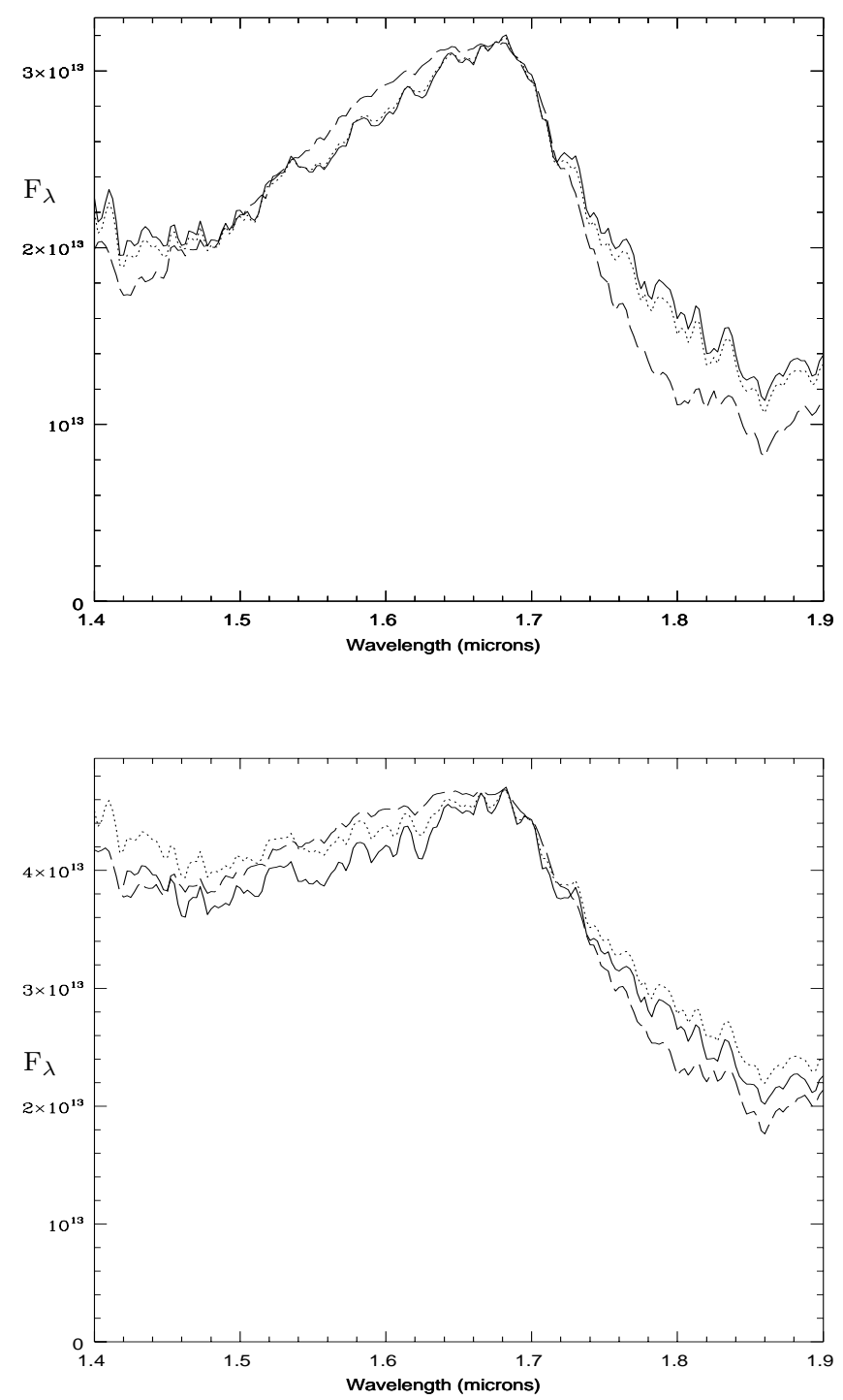

Figure 12: Effect of gravity on AMES-Dusty models at $H\left(\mathrm{~F}_{\lambda}\right.$ spectra). (top) at $2500 \mathrm{~K}$ the $[\mathrm{g}]=3.5$ spectrum (solid line) and $[\mathrm{g}]=4.0$ spectrum (dotted line) are very similar but the $[\mathrm{g}]=5.5$ model (dashed line) has a flatter peak. (bottom) at $2900 \mathrm{~K}$ the $[\mathrm{g}=3.5]$ and $[\mathrm{g}=4.0]$ spectra begin to diverge. The $[\mathrm{g}=5.5]$ spectum requires a lower temperature, $2800 \mathrm{~K}$, to produce similar water absorption and crosses the lower gravity spectra.

$0.119 \pm 0.055$ for $055-231$, consistent with $K_{C O}=0.12$ at $\mathrm{T}_{\text {eff }}=2575 \mathrm{~K}$ (with $\left.\log (\mathrm{g})=3.5\right)$ but not $K_{C O}=0.21$ (with $\log (\mathrm{g})=5.5)$. Similarly, we observe $K_{C O}=0.059 \pm 0.035$ for 096-1944, roughly consistent $K_{C O}=0.106$ at $\mathrm{T}_{\text {eff }}=2700 \mathrm{~K}$ $(\log (\mathrm{g})=3.5)$ but not $K_{C O}=0.167(\log (\mathrm{g})=5.5)$. The model predictions for $\log (\mathrm{g})=3.5$ and $\log (\mathrm{g})=4.0$ are almost identical.

In 6/11 sources $\mathrm{CO}$ is not unambiguously detected in these low resolution spectra, since the drop in flux seen in all sources at $\lambda>2.3 \mu \mathrm{m}$ may be due to $\mathrm{H}_{2} \mathrm{O}$ as well $\mathrm{CO}$, and the bandheads are not sufficiently resolved. We place upper limits on the $K_{C O}$ index for these sources in Table 3. Again these are consistent with low gravity models, but not with high gravity models in the case of the cooler sources, since CO depths of order $20 \%$ would be detected. This strong 


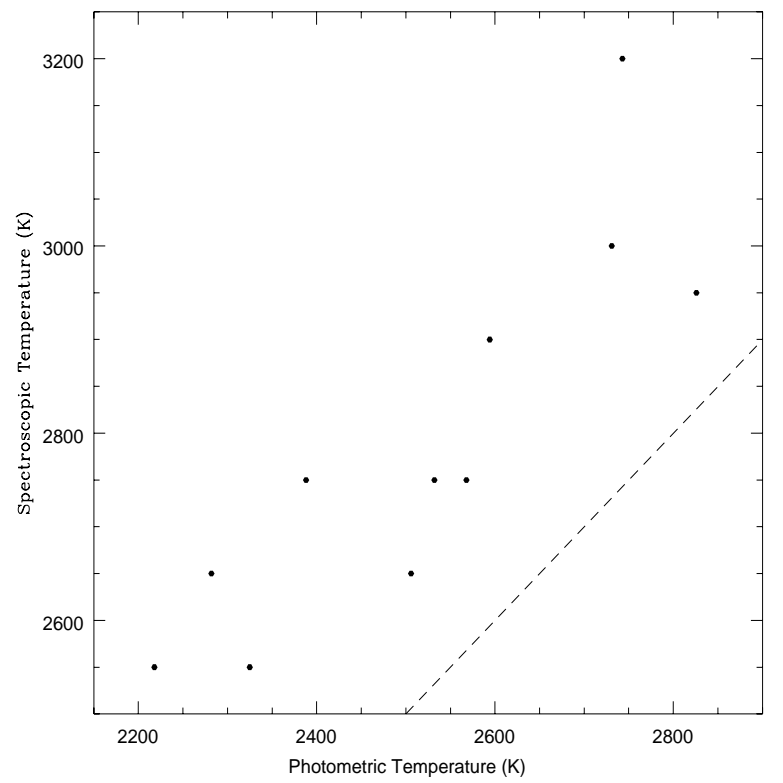

Figure 13: Spectroscopic Temperatures $[\mathrm{g}=4.0]$, correlated with independent Photometric Temperatures for sources with measured (dereddened) (I-J) colours. The correlation is excellent but the spectroscopic temperatures are higher by $250 \mathrm{~K}$, as indicated by the dashed line for perfect agreement.

absorption is observed in local dwarfs, as evidenced by our spectra of 3 red standards in Figure 16.

In field dwarfs a fairly strong feature is often observed near $1.62 \mu \mathrm{m}$, with uncertain identification at present. The AMES-Dusty-1999 spectra resolve this into a complex feature with possible contributions from several lines of $\mathrm{OH}$, $\mathrm{CaI}$ and $\mathrm{FeI}$, with an $\mathrm{OH}$ doublet at $1.624 \mu \mathrm{m}$ predicted to dominate in low gravity atmospheres. Giant spectra show the $(\mathrm{v}=6-3)$ CO bandhead at $1.619 \mu \mathrm{m}$ in low resolution spectra, which increases in strength in very low gravity supergiants (Origlia, Moorwood \& Oliva 1993), but this is not predicted to be important at $\log (g)=3.5$ to 4.0. An absorption feature is marginally detected at the $1.624 \mu \mathrm{m}$ wavelength in the composite Orion spectrum in Figure 5(a-b) but it is not unambiguously distinguished from noise and is much weaker than the $\log (g)=3.5$ models indicate for the $\mathrm{OH}$ dominated features at $\mathrm{T}_{\text {eff }} \geq 2800 \mathrm{~K}$. At $\log (g)=4.0$ the $1.624 \mu \mathrm{m}$ feature is no stronger than at $\log (g)=5.5$, for the same temperature, so at that gravity the absence of a clear detection would be consistent with the data. (The AMES-Dusty-1999 grid has a sampling interval of 0.5 dex in gravity).

Considering that the $\log (g)=4.0$ models also produce fitted spectroscopic temperatures more consistent with the evolutionary tracks than $\log (g)=3.5$ models, this might be taken as evidence that the gravities are closer to the higher value, though a finer grid in gravity would be needed to demonstrate this. On the BM97 1 Myr isochrone the $3.5<\log (g)<4.0$ interval covers an age range from $<0.1$ Myr to several Myr for $0.008 \mathrm{M}_{\odot}$ planetary mass objects, so a very precise gravity measurement would be needed to determine the age with useful precision. A slightly greater than average age might be expected for this spectroscopic sample of sources with low extinction.

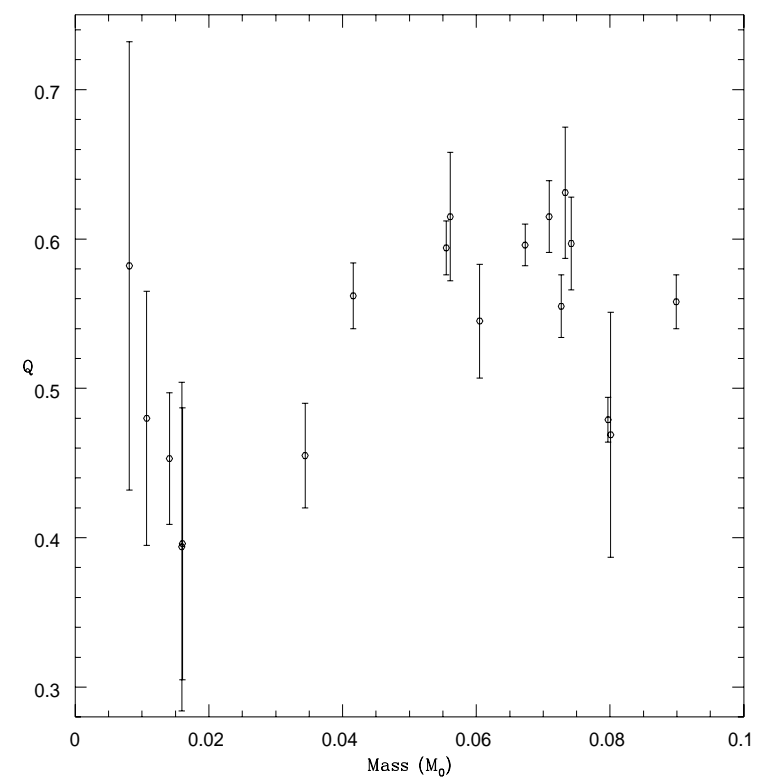

Figure 14: Spectroscopic Temperature $[\mathrm{g}=4.0]$ vs. Mass. Masses are from the BM97 1 Myr isochrone. A weak correlation is observed and the temperatures are consistent with the essentially independent BM97 prediction.

\subsubsection{Atomic Lines}

The absence of the NaI line at $2.21 \mu \mathrm{m}$ in $053-503$ (our best $K$ band spectrum) is also indicative of a low gravity atmosphere, as shown by the AMES-Dusty-1999 spectra in Figure 15. 053-503 shows only a weak local minimum at the 1- $\sigma$ level: this cannot be distinguished from noise but is consistent with the model. The weakness of NaI at $2.21 \mu \mathrm{m}$ has been observed in a low gravity YSO by Luhman et al.(1998b). In addition, the weakness of optical $\mathrm{NaI}$ and KI features in very young brown dwarfs has been observed by Bejar et al.(1999) in the $\sigma$-Orionis cluster; by Luhman, Liebert \& Rieke (1997) in a brown dwarf in the $\rho$ Ophiuchus region; and by Briceno et al.(1998) in pre-main sequence stars and brown dwarfs in Taurus.

\subsection{Comparison with $\sigma$-Orionis Spectra and IC348 data}

We note that the $H$ and $K$ bandpass spectra of 2 very low mass candidates in the $\sigma$-Orionis cluster (estimated age $\sim 5 \mathrm{Myr}$ ), plotted as low signal to noise smoothed spectra by Zapatero-Osorio et al.(2000), do not resemble those presented in Figure 4(a-c). At $H$ they show the plateau seen in field dwarfs and they also appear to be redder in the ratio of $K: H$ bandpass fluxes than all of the sources plotted in Figure 8(a-b) with strong water absorption, except 014-413, which appeared to have an infrared excess due to hot circumstellar dust. After converting the spectra to $\mathrm{F}_{\nu}$ the apparent ratios of flux near the bandpass peaks are $K: H \approx$ 1.6 and 1.2 for S Ori 47 and S Ori 60 respectively. The presence of the plateau feature might be taken as evidence that these $2 \sigma$-Orionis objects are field $\mathrm{L}$ dwarfs, given that Zapatero-Osorio et al. expect $\sim 33 \%$ contamination by these in their much larger survey area. However, there is separate evidence for the relative youth of 1 of the 2 , S Ori 47, 

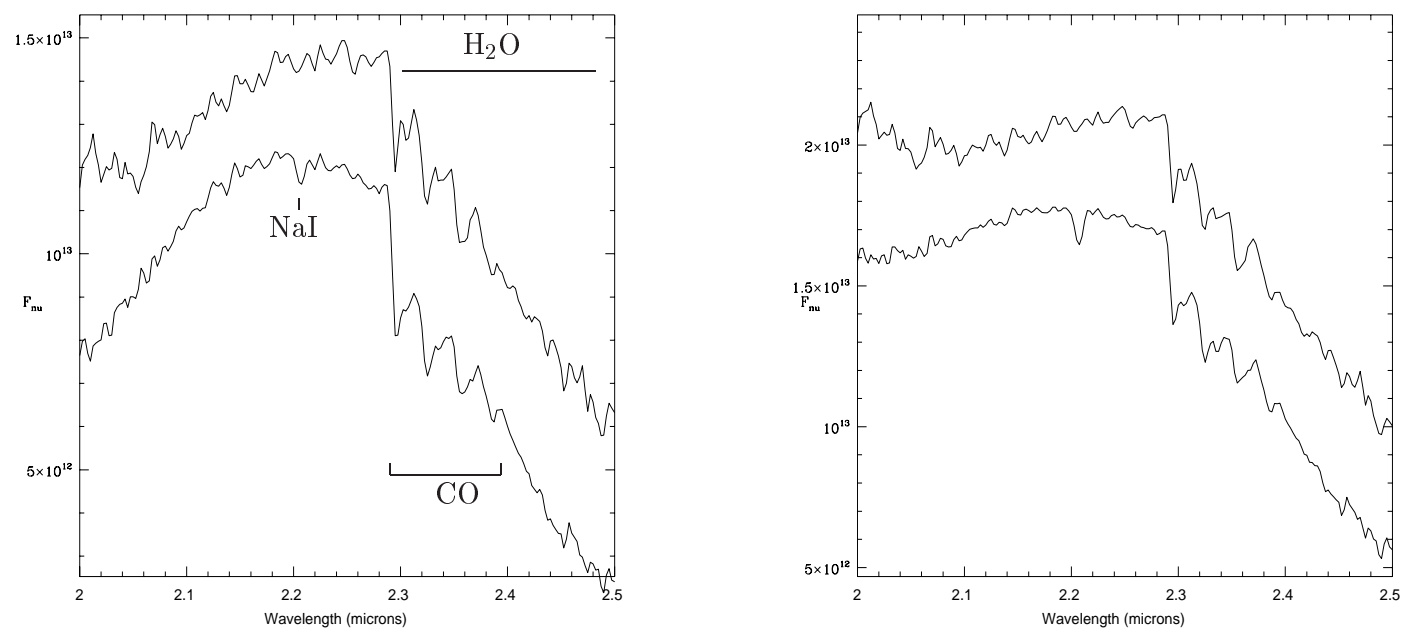

Figure 15: Gravity effects on $\mathrm{CO}$ and $\mathrm{NaI}$ ( $\mathrm{F}_{\nu}$ spectra). (left) $2500 \mathrm{~K}$; (right) $2900 \mathrm{~K}$. Both $\mathrm{CO}$ and NaI are much stronger in the low gravity $[\mathrm{g}=3.5]$ AMES-Dusty spectra (offset lower plots) than the $[\mathrm{g}=5.5]$ atmospheres. $[\mathrm{g}=3.5]$ and $[\mathrm{g}=4.0]$ are nearly identical at these wavelengths.
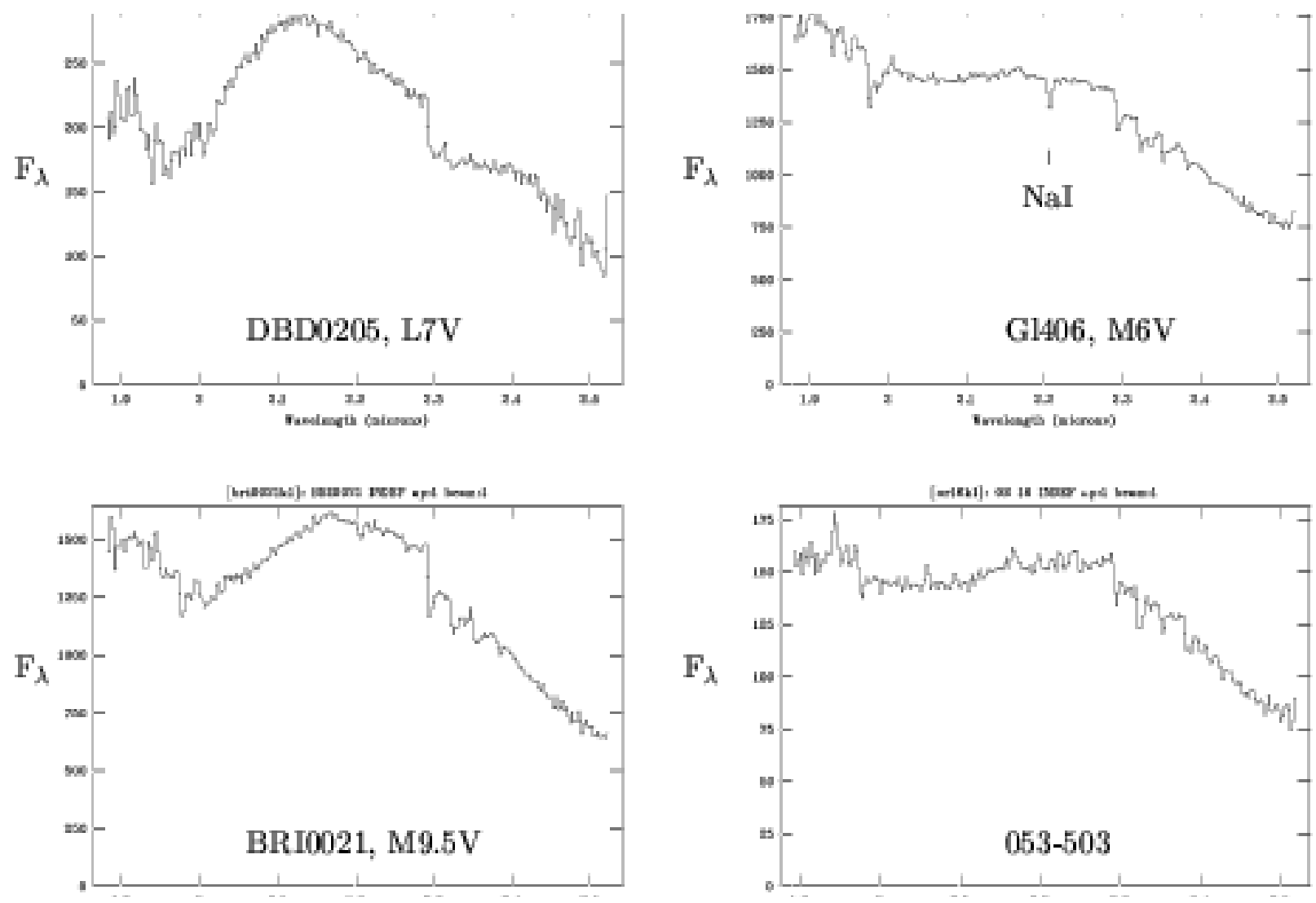

Figure 16: Field Dwarf spectra and 053-503. Late M and L type spectra of high gravity field dwarfs show very strong CO. 053-503 has similar $\mathrm{CO}$ to the M6 field dwarf but shows no NaI and stronger water absorption. 
via low gravity features in its optical spectrum (ZapateroOsorio et al. 1999) similar to those seen in Pleiades dwarfs. It would have to be an unusually young $(\sim 100 \mathrm{Myr})$ field $\mathrm{L}$ dwarf to have those optical features. In our view it is more likely that the $H$ bandpass spectra and larger $K: H$ ratios reflect the greater age of the $\sigma$-Orionis cluster than the Trapezium cluster. At $5 \mathrm{Myr}$, sources at or below the deuterium burning threshold are predicted to be $300 \mathrm{~K}$ or $500 \mathrm{~K}$ cooler than at $1 \mathrm{Myr}$ (Baraffe et al;BM97 calculations respectively), corresponding to an effective temperature $\left(\mathrm{T}_{\text {eff }}\right)$ of $1600-1800 \mathrm{~K}$ for an object with mass $8 \mathrm{M}_{J u p}$ and $\sim 2200 \mathrm{~K}$ at $15 \mathrm{M}_{\text {Jup }}$ according to both calculations. This is substantially cooler than the Trapezium sources and similar to the temperature of L type field dwarfs which exhibit the plateau feature. This is well into the $\mathrm{T}_{\text {eff }}<2500 \mathrm{~K}$ region in which dust in the photosphere starts to have a large influence upon the emergent spectrum, according to the AMES-Dusty-1999 models. The larger ratio of $K: H$ band flux is also predicted for a lower temperature, being caused by the underlying Planckian shift of flux to longer wavelengths rather than circumstellar dust. There is a some indication of a plateau feature in the AMES-Dusty models at $2200 \mathrm{~K}$ in the $1.64-1.68 \mu \mathrm{m}$ region (not shown) but it is much less broad than that observed by (Zapatero-Osorio et al.(2000) and weakens at lower temperatures. This might be due either to difficulties with the treatment of water or dust in the model or differences in the stratification of dust at this very early evolutionary stage. We emphasise that our $H$ and $K$ bandpass spectra of 3 field dwarf standards in Figure 5 and Figure 16 are in excellent agreement with the prior observations of Leggett et al.(2001), Jones et al.(1994), Tokunaga \& Kobayashi and Delfosse et al. so we have no reason to doubt the reliability of the Trapezium spectra. Scattering in the circumstellar environment might affect the spectral profiles in subtle ways but would not erase features at specific wavelengths such as the $H$ bandpass plateau.

Najita, Tiede \& Carr (2000) have discovered 6 sources at or below the deuterium threshold in the very young cluster IC348 (typical age $3 \mathrm{Myr}$ ), extending the work of Luhman et al. (1998a). Although they did not have spectra, they were able to obtain approximate spectral types via narrow band imaging across the $1.9 \mu \mathrm{m}$ water band with NICMOS on the Hubble Space Telescope. They came to very similar conclusions to those presented here: the water absorption strength implied late $\mathrm{M}$ or $\mathrm{L}$ spectral types and they also argue that their sources lie above the main sequence and are therefore not background stars. This foreshadows our argument in the next section. An interesting difference is that according to the AMES-Dusty models their $1.9 \mu \mathrm{m}$ water index is stronger in low gravity pre-main sequence objects than in high gravity field stars of the same temperature. This apparent contrast with our earlier statement is due to the different broad band profiles of low and high gravity objects. The behaviour of water absorption strength as a function of gravity depends on the wavelength at which the water absorption is measured. We will have to wait for the NICMOS Cooling System to be installed before measurements such as those of Najita et al. can be taken elsewhere, since telluric water absorption generally precludes good quality ground based observation at these wavelengths.

\section{CLUSTER MEMBERSHIP}

The reality of the population below $0.02 \mathrm{M}_{\odot}$ has been called into question by Hillenbrand \& Carpenter (2000), who suggest that lightly reddened background field dwarfs are present in the data, and are not distinguished from the proposed planetary mass population. They estimated that the total contamination by non-cluster members is $5 \%$ to $\mathrm{K}=18$, using a modified Wainscoat model (Wainscoat et al. 1992) of the entire galactic stellar population. This type of calculation is difficult, owing to substantial uncetainties in: (a) the infrared extinction on lines of sight through the cluster; (b) the scale height of the galactic $\mathrm{M}$ and $\mathrm{L}$ dwarf populations. This is relevant because the Trapezium lies $\approx 122 \mathrm{pc}$ above the plane, relative to the sun, which is more than 1 scale height above the Young Disk population but probably not the Old Disk.

To address this issue we first look at the spectroscopic data to see whether they are consistent with a field dwarf population. Five dereddened $H$ bandpass spectra of sources with 1 Myr masses $\mathrm{M} \leq 0.016 \mathrm{M}_{\odot}$ and $0<A(V)<2.3$ are shown in Figure $4(\mathrm{c})$. Although the signal to noise ratio (SNR) in these spectra is low, it is clear that all possess deep water absorption on either side of the peak near $1.68 \mu \mathrm{m}$, which would correspond to L-type spectra if they were main sequence dwarfs (see Table 3 ). The spectral types are uncertain by a few subtypes due to the low SNR and the possibility of imperfect background subtraction but they are derived by a reddening independent method, so extinction does not affect the typing. Alternative water-based derivations of spectral type could lead to earlier classifications, e.g. the Delfosse slope-based water index (see Section 4) which samples the steepest part of the water absorption in field dwarfs and not the plateau. However, we are confident that the $H$ bandpass spectra of the very low mass candidates are of type M7 or later by any logical definition. For example, spectra of M0-M6 dwarfs do not decline significantly in flux/pixel (i.e. $\mathrm{F}_{\lambda}$ ) on the short wavelength side of the $H$ bandpass, going from 1.68-1.5 $\mu \mathrm{m}$ (see eg. Leggett et al.2000, 1996), whereas the very low mass candidates all do, as illustrated in Figure 17 for the candidates with weakest water absorption. Early M-type spectra rise from 1.7 to $1.25 \mu \mathrm{m}$, since water absorption is weak at these types in Disk population dwarfs and even weaker in Halo extreme subdwarfs.

On the CIT photometric system an M7V dwarf has an absolute magnitude $\mathrm{M}_{H}=10.22$, according to a fit to sources with known parallaxes by Leggett (1992); or $\mathrm{M}_{H}=10.26$, averaging 2 sources from Leggett et al.(2001) with measured parallaxes in the Yale Catalogue (van Altena et al. 1995). Adopting the brighter value, this leads to $\mathrm{m}_{H}=18.44$ for a source just behind OMC- 1 at $440 \mathrm{pc}$, or $\mathrm{m}_{H}=18.57$ on the UFTI magnitude system. This is more than a magnitude less luminous than the 3 very low mass brown dwarfs in Figure 4(c) and 0.6 to 0.9 magnitudes less luminous than the 2 planetary mass candidates (with $\mathrm{H}_{d r}=17.71,17.95$ on the UFTI system). Uncertainties in the dereddened $H$ band fluxes for these 5 sources are expected to be $\approx 0.2$ magnitudes via the random photometric error and 0.15-0.2 magnitudes possible systematic error due to the uncertainty in the $(J-H)$ colour of the isochrone. Later type dwarfs decline rapidly in brightness, eg. $\mathrm{M}_{H}=10.90$ at M9, and 10.98 at L0 on the CIT system, as fitted by 

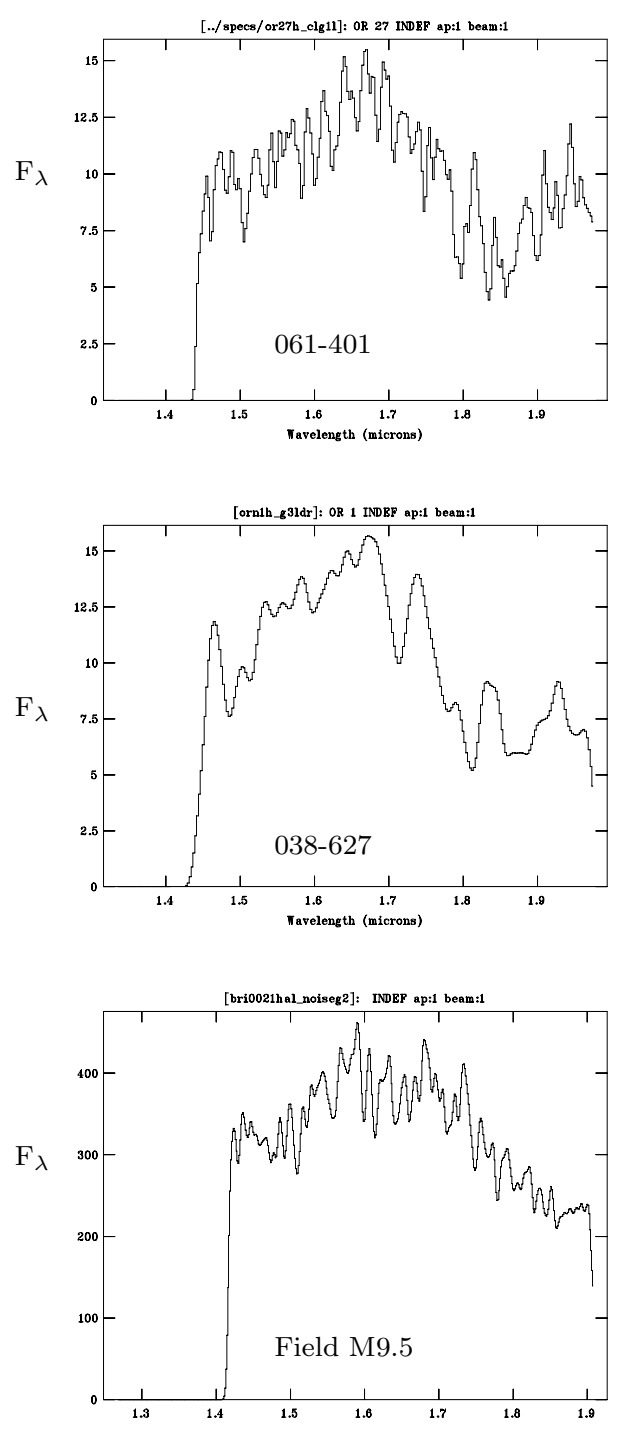

Figure 17: $\mathrm{F}_{\lambda}$ spectra of 061-401 $\left(0.011 \mathrm{M}_{\odot}\right)$ and 038-627 $\left(0.014 \mathrm{M}_{\odot}\right)$ compared with field dwarf BRI0021-0214. The field dwarf spectrum is given added noise and smoothed for comparison.

Kirkpatrick et al.(2000) using sources with measured parallaxes. Hence, our very low mass sources are almost certainly too luminous to be background main sequence dwarfs, even assuming zero extinction by the OMC-1 cloud. In fact, the backdrop of the OMC-1 cloud provides substantial extinction in the region of the spectroscopic sample, as discussed below, which further reinforces this result. The luminosities are higher because they are physically much larger and have higher effective temperatures than field dwarfs with equivalent water absorption depths.

The profiles shown in Figure 4(c) also resemble those of higher mass Trapezium objects rather than that of a late $\mathrm{M}$ or L type field dwarf, illustrated in Figure 17 with noise added to aid the comparison. The field dwarf has a plateau from 1.57-1.70 $\mu \mathrm{m}$, whereas the very low mass candidates appear to peak near $1.68 \mu \mathrm{m}$ like their brighter counterparts.

If background contamination were significant we would expect a range of lightly reddened M0-M6 dwarfs to appear in our sample, since these are the commonest stars which are luminous enough to be detected at $d \geq 400 p c$, assuming very low extinction by OMC-1 in the outer regions of our survey. The earlier M-types are less common than the later types but would be detectable to a greater distance, so the distribution would be fairly even across spectral types M0-M6. Since they are not seen among our planetary mass candidates we suspect that $\mathrm{HC}$ have underestimated the extinction for 2 reasons. The first reason is that the extinctions derived from our $(J-H)$ colours show that the $\mathrm{A}(\mathrm{V})$ column extends beyond the spatial pattern indicated by $\mathrm{C}^{18} \mathrm{O}$ maps, which are only sensitive to high density gas. High extinction (up to $\mathrm{A}(\mathrm{V})=14$ ) is measured toward many sources at the edges of our survey (see Figure 18), which we attribute mainly to lower density molecular gas and also to the neutral cluster medium (O'Dell \& Yusef-Zadeh 2000). The near infrared extinction measurements are direct, whereas values derived from radio measurements are always uncertain by a factor of a few. Nevertheless, there is radio waveband confirmation of the extinction. The ${ }^{13} \mathrm{CO}$ and $\mathrm{CS}(1-0)$ maps of Tatematsu et al.(1993) sample the less dense molecular gas and show that the OMC-1 cloud has a low density wing extending far to the west of the main ridge in the cluster centre, beyond the location of our sources. The column density of the wing corresponds to $\mathrm{A}(\mathrm{V}) \approx 4$ in the outer parts of the wing (using the transformations of Lada et al. 1994) for ${ }^{13} \mathrm{CO}$ and may be higher in the inner part where our sources lie: this region is not well resolved spatially from the very high density ridge in the map of Tatematsu et al. In addition, the cold dust is seen directly in the $850 \mu \mathrm{m}$ SCUBA data of Johnstone \& Bally (1999), who measure continuum emission of $0.5-2.0 \mathrm{Jy} /$ beam in the spectroscopic survey region. Applying their adopted emissivity index $(\beta=2)$, temperature $(20 \mathrm{~K})$ and 100:1 ratio of gas:dust, we calculate 2$8 \times 10^{29} \mathrm{Kg}$ per beam (14 arcsec diameter). Adopting the relation $\mathrm{A}(\mathrm{V}) / \mathrm{N}(\mathrm{H})=5.3 \times 10^{-26} \mathrm{~m}^{2}$ leads to $4.8<A(V)<19$ in the survey region, where the very low mass sources are detected. The uncertainties in grain emissivity, temperature and $\mathrm{A}(\mathrm{V}) / \mathrm{N}(\mathrm{H})$ mean that this is only an order of magnitude estimate (eg. Ward-Thompson et al.1994) but it is consistent with the near infrared and ${ }^{13} \mathrm{CO}$ data. The second reason to expect significant extinction is that $\mathrm{HC}$ used an optical:infrared extinction conversion based on the interstellar medium, which significantly underestimates the infrared extinction in Orion (Cardelli et al.) because of the presence of slightly larger dust grains in the environment of the molecular cloud. The substantial contribution of these slightly larger grains to extinction can be seen from calculations using Mie theory (eg. Figure 15 of Lucas \& Roche 1998). In some very young sources the measured infrared extinction may be local to the young star. However, comparison of Figure 18 and Figure 3 does not show any less extinction in the outer region of our survey than in the full survey sample, which indicates that there is strong extinction by the low density molecular gas throughout the survey region. Moreover, the $L$ bandpass data of Lada et al.(2000) show that very young, embedded protostars are strongly concentrated towards the high density ridge, so the case for significant extinction by lower density gas in the outer cluster is clear. It therefore appears that any background stars of type M7 or later would appear more than a magnitude fainter than all 


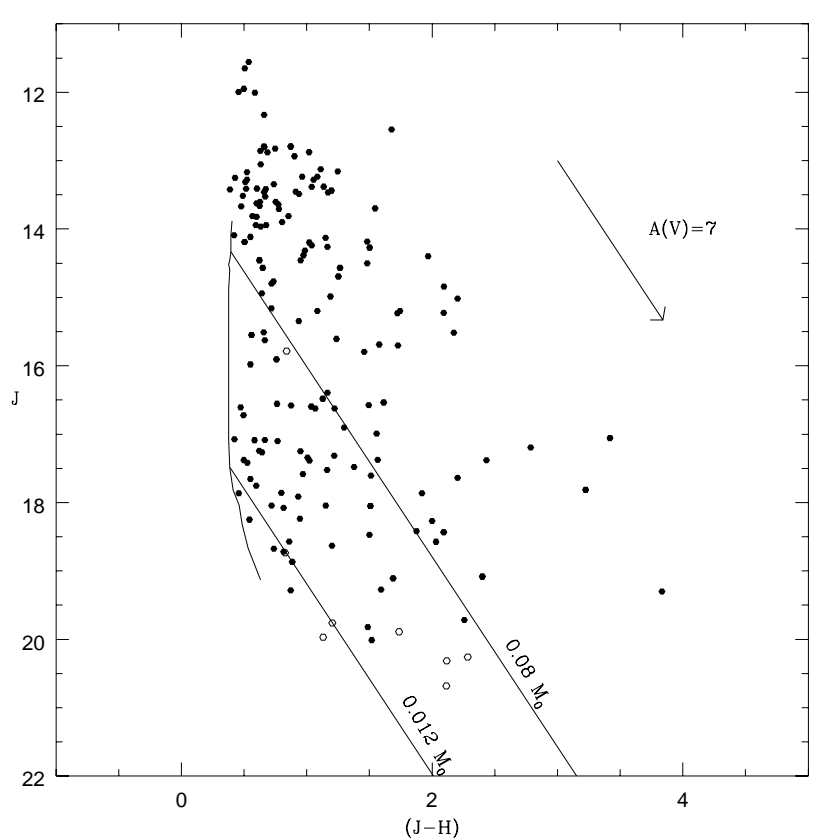

Figure 18: Colour magnitude plot for the region of the spectroscopic sample. High reddening is observed toward many many sources, similar to Figure 3. The new Baraffe et al. isochrone is plotted at left.

the low mass candidates in Figure 4(c) and even background binaries would not be bright enough to cause confusion.

Our assumption in Paper I that the OMC-1 cloud prevented any background contamination in the survey region at $J$ band may prove to be an overstatement however. In Section 3.2.1, we discussed the 2 candidates near the stellar/substellar boundary (019-108, 066-433) in our spectroscopic sample which suffer high extinction $(\mathrm{A}(\mathrm{V})=8.6,12.6$ respectively) and have weak water absorption, consistent with late K-type or very early M-type spectra. If they are embedded YSOs then we can say that there is no background contamination in our sample, which is highly encouraging for future work in this cluster. It is very possible that they are background field stars, since their fluxes are consistent with that interpretation and the extinction is broadly consistent with what we might expect from the radio data. This would also be encouraging, since the high extinction towards these 2 sources would indicate that background contamination can be avoided by studying sources which have low extinction.

In Paper I we had assumed that lightly reddened background sources are not present because of the large near infrared extinction of cluster members and because the cluster profile at infrared wavelengths is apparently identical to that at visible wavelengths. A large population of field sources would be expected to produce a broader profile at infrared wavelengths, since these sources are uniformly distributed and more easily seen at the edges of the cluster, where extinction is lower. This argument was first used by Hillenbrand \& Hartmann (1998) but not at such faint sensitivity limits. We apply it here using our $I$ and $H$ bandpass data, in Figure 19, plotting the number of detections in each filter as a function of distance from $\theta_{1}$ Ori C. Inspection of the profile gives no hint of a background population since the ratio of $H: I$ bandpass detections is fairly uniform and may even decrease slightly between radii of 200 and 300 arcsec. This

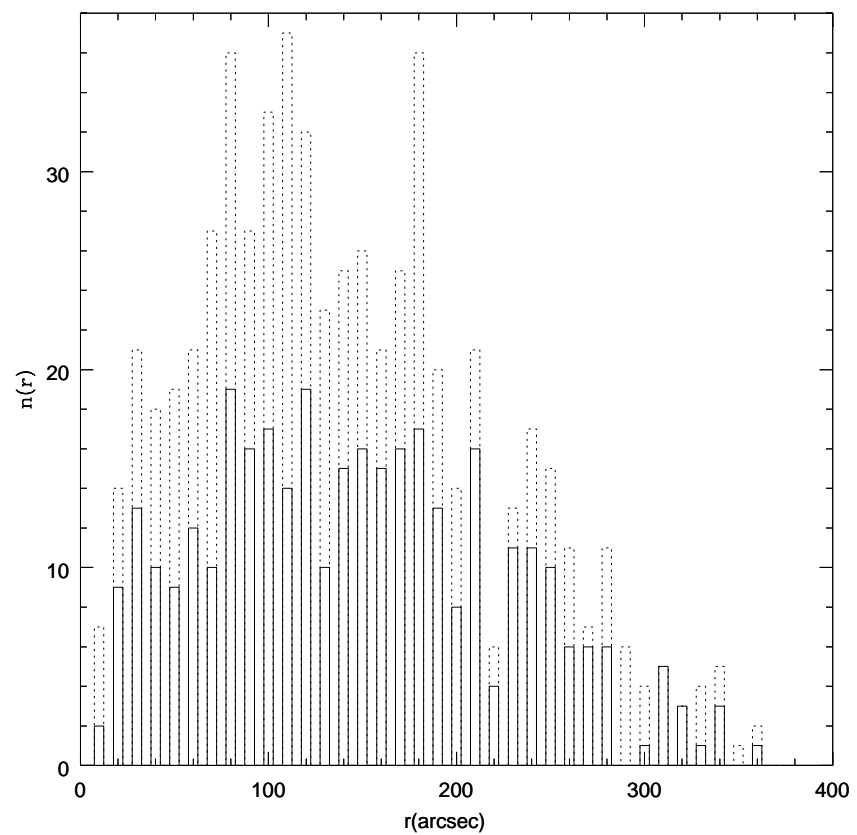

Figure 19: Trapezium Cluster profile $\mathrm{n}(\mathrm{r}) \mathrm{dr}$ as a function of distance from $\theta_{1}$ Ori C. Solid lines show $I$ band detections, dashed lines show $H$ band detections. The profile is incomplete beyond 160 arcsec because the survey region is not circular, but extends to the west.

demonstrates that any population of faint sources $(H \gtrsim 17$ to be undetected at $I$ ) must be small in order to have no observable impact on the cluster profile. This method is not precise enough to exclude the presence of a small background population and there are selection effects in Figure 19 because the detectabilty of sources increases more rapidly at large radii in the $I$ bandpass than at $H$, owing to the greater effect of the nebular background at $I$. Nevertheless, the absence of any obvious background population in the profile at $H$ suggests that contamination is likely to be small at $J$.

Could the planetary mass objects be foreground dwarfs? About half of the 15 candidates have sufficient reddening, $(J-H)>0.85$ (UFTI system) or $(J-H)>0.94$ on the CIT system, for us to be confident that they are significantly reddened. Only field dwarfs of type L2 and later can reach these colours according to Kirkpatrick et al.(2000) (or L6 and later according to the smaller sample of Leggett et al. 2001). These provide very small contamination, since mid and late L-types are only detected at small distances, sampling a much smaller volume than late M-types $\mathrm{m}_{J}=20.0$ ). Kirkpatrick et al.(2000) The expected number of foreground objects is only 5 or 6 in the full survey region ( $\sim 1 \%$ contamination) according to both the Tinney et al.(1993) luminosity function and the 2MASS data (Reid et al.1999), mainly M dwarfs in magnitude range $15<J<19$. We expect to detect $\leq 1$ foreground L dwarf in the full survey region of $40 \operatorname{arcmin}^{2}$, using the space density estimate of ZapateroOsorio et al.(2000). Although the volume density of field L dwarfs is uncertain, the number cannot rise by more than a factor of 3, since Zapatero-Osorio et al. calculated 33\% contamination in their much larger survey area at very similar galactic coordinates (contamination cannot exceed 100\%). Planetary mass candidates $\left(\leq 0.012 \mathrm{M}_{\odot}\right)$ are detected in no more than $60 \%$ or our survey area, where the nebular sur- 
face brightness is sufficiently low. Hence, we derive an upper limit of 1 or 2 late $\mathrm{M}$ and $\mathrm{L}$ type sources which could appear to be planetary mass objects.

\section{AGE AND MASS OF SOURCES}

The interpretation of very faint cluster members as planetary mass objects might be incorrect if they are far older $(\geq 20 \mathrm{Myr}$ ) than the bulk of the higher mass cluster members. The luminosity of a $0.020 \mathrm{M}_{\odot}$ object does not decline to that of the 6 candidates with $\mathrm{M} \lesssim 0.008 \mathrm{M}_{\odot}$ until an age of $27 \mathrm{Myr}$, according to the BM97 calculations. The age distribution of the population has been studied in detail by Hillenbrand (1997) over the wider Orion Nebula Cluster, within a radius of 20 arcmin, using 2 evolutionary tracks in the HR diagram. This analysis concluded that most star formation in Orion has occurred within the past $2 \mathrm{Myr}$ and that the youngest sources are highly concentrated towards the cluster centre, which is the region covered by our relatively small scale survey. The dataset did not include planetary mass sources but indicated that the lowest mass and highest mass sources are most strongly concentrated toward the cluster centre, with less concentration by intermediate (solar mass) sources. Specifically, Hillenbrand calculated that $<2 \%$ of the population has an age $>20 \mathrm{Myr}$, using the D'Antona \& Mazzitelli (1994) tracks, which agree well with more recent calculations (BM97; D'Antona \& Mazzitelli 1998, Baraffe et al.). The Swenson et al.(1994) tracks, which indicated lower temperatures for brown dwarfs than more recent calculations, put the proportion at $\sim 5 \%$, which gives some indication of the uncertainty in this type of calculation. Conservatively, we assume that the old ( $>20 \mathrm{Myr})$ population is only a factor of 3 less concentrated within our survey region (radius $\approx 4$ arcmin) than the young population (see Figure 22 of Hillenbrand 1997). This yields a contamination of $0.6 \%-1.7 \%$ by such old objects in our sample, or 4 to 11 objects spread over all masses in the sample of $\sim 650$ sources detected at both $J$ and $H$ (including bright saturated sources). The more recent assessment of the age distribution in the core of the cluster by $\mathrm{HC}$ gave a similar result. Expressed as $\mathrm{N}(\log (M))$, the IMF in Orion has been demonstrated to have a broad maximum somewhere between 0.1 and $0.6 \mathrm{M}_{\odot}$ (Paper I, HC, Luhman et al.2000) and decline slowly at substellar masses. In the 0.03-0.08 $\mathrm{M}_{\odot}$ interval a relation $\mathrm{N}(\log (M)) \propto M^{-1}$ is consistent with our data in Paper I. If this trend continues below our completeness limit to $0.013 \mathrm{M}_{\odot}$, then we would expect there to be $\sim 0.16$ to 0.44 old brown dwarfs with sufficiently low mass $(\sim 0.013$ to $0.03 \mathrm{M} \odot)$ to masquerade as a planetary mass object. Even if the substellar IMF were flat we would not expect there to be more than 1 such source, whereas there are $\sim 15$ planetary mass candidates. In the event of a flat substellar IMF it would be difficult to argue for an abrupt cut off above the deuterium threshold.

We note that the status of 1 brown dwarf candidate, 014-413 as a youthful Trapezium cluster member has been confirmed by direct imaging of a wind bow shock interaction with the cluster medium in $\mathrm{H} \alpha$ in a Hubble Space Telescope survey (Bally, O'Dell \& McCaughrean 2000) which unfortunately overlaps only a small part of the spectroscopic survey region. Outflow activity is correlated with accretion rate in
YSOs, which decreases with age. Hence, this is probably a younger than average cluster member, which is also indicated by the presence of a large $K$ bandpass excess in Figure $8(\mathrm{a})$.

$K$ bandpass excesses are a good indicator of youth, since they are most common at ages $\leq 1 \mathrm{Myr}$. Is there evidence for $K$ bandpass excesses in our planetary mass candidates ? $6 / 15$ of our planetary mass candidates were detected in the $H$ and $K$ bandpass data of $H C$, which only partially overlaps our survey area. These can be dereddened with the addition of our $J$ bandpass data. The $H$ bandpass fluxes measured by $\mathrm{HC}$ agree well with ours in $3 / 6$ cases, the measurements differing by $\leq 0.13$ magnitudes, see Table 5 . We note that the $3 / 6$ sources with poor agreement all lie near the edge of the survey of $\mathrm{HC}$, and are believed to suffer from substantially shorter exposure times ( $25 \mathrm{~s}$ or $50 \mathrm{~s}$ total), whereas the other 3 are believed to have the full $100 \mathrm{~s}$ exposure time. The poor agreement is therefore probably due to low SNRs in the HC data, given that all these faint sources are near the sensitivity limits of both surveys. Alternatively, large amplitude variability may occur in a small minority of sources, which has been demonstrated at stellar mass by Carpenter, Hillenbrand \& Skrutskie (2001). If we accept that the 3 sources with good agreement have well measured $H$ bandpass fluxes, then there appear to be large $K$ band excesses in $2 / 3$ cases. It is risky to compare photometry from different surveys in this way but these colour excesses, $\mathrm{E}(\mathrm{H}-\mathrm{K}) \approx 0.6$ magnitudes, are large enough to be fairly convincing despite the uncertainty of order $0.1 \mathrm{mag}$ in the $(J-H)$ colour of the photospheres. If they are genuine then this indicates that these are very young sources, probably with ages of $<1 \mathrm{Myr}$, and therefore are very good planetary mass candidates.

Oasa et al.(1999) were cautious in interpreting very faint sources in the Chamaeleon I cloud as planetary mass objects, despite the detection of large $K$ bandpass infrared excesses requiring the presence of a substantial mass of hot dust in the accretion disk. However they found that even at $10 \mathrm{Myr}$, their presumed upper age limit for sources with substantial quantities of hot dust, a planetary mass was still indicated for their faintest source.

At present, there is no easy way to actually measure the ages of individual sources because luminosity and temperature are degenerate with respect to mass and age, and because of the need to deredden sources. In principle the tracks of more massive sources might be separated on an HR diagram (older, more massive sources should be smaller and hotter than planetary mass objects of equal luminosity). At present, however the uncertainty in temperature measurement and prediction at these very early ages is too large to make such a distinction (see Table 3). A small bias toward greater ages might be expected for the spectroscopic sample of sources with low extinction (see Section 4.3). Precise measurements of surface gravity via high resolution spectroscopy of absorption line profiles may permit this at some future date but this will be difficult owing to the weakness of atomic lines in low gravity atmospheres. A promising alternative would be to use well modelled lines of simple molecules such as $\mathrm{CO}$, and perhaps the $\mathrm{K}_{C O}$ index, because some molecular features increase in strength with decreasing gravity. Another option might be to search for deuterium, the defining attribute of these 'free floating planets'. Deuterium is predicted to be absent in sources more $10^{7}$ years 
old, for masses $\mathrm{M}>0.03 \mathrm{M}_{\odot}$. This is illustrated by Bejar et al., using the calculations of D'Antona \& Mazzitelli (1997), and examined in more detail by Chabrier, Baraffe, Allard, \& Hauschildt (2000).

\section{GEMINI DATA}

We have conducted a pencil beam search for sources in the Trapezium even fainter than those deetected in our UKIRT survey, using the Gemini North Telescope with the Adaptive Optics system Hokupa'a and the infrared camera QUIRC. The data will be described more fully in a future paper but preliminary indications are that no such ultra low luminosity sources were detected in 5 QUIRC fields containing $\sim 25$ stars and brown dwarfs. This tends to support our previous suggestion thet the Luminosity Function (LF) in the Trapezium turns down somewhere near $8 \mathrm{M}_{J u p}$, but this is far from conclusive because of the very small area surveyed and the existence of small scale spatial variation in the IMF, as seen in Figure 2). The decline of the LF should be investigated further to see whether it is a feature of the IMF or merely of the mass-luminosity relation. (An attempt at a much larger companion survey with the NAOMI Adaptive Optics system at the William Herschel Telescope was prevented by severe weather.) There are theoretical arguments for expecting few sources below $10 \mathrm{M}_{\text {Jup }}$ (Silk 1977; Adams \& Fatuzzo 1996) but the physical processes in crowded clusters and multiple star systems may circumvent the obstacles described in those papers.

\section{CONCLUSIONS}

Low resolution $H$ and $K$ bandpass spectra of brown dwarfs and planetary mass candidates show strong water absorption bands, equivalent in depth to those seen in L-type and late M-type spectra, which is entirely consistent with the predictions of evolutionary tracks (Baraffe et al., BM97, DM98) at ages near 1 Myr. The new evolutionary tracks provide self consistent flux predictions, permitting luminosities and masses to to be derived without the need for bolometric corrections. The results are very similar to our previous work. However, the $H$ bandpass profiles shown here are very different from those of field dwarfs with strong water absorption, which appears to be a clear signature of youth and low mass. Strong evidence for the expected low surface gravities is provided by fitting the spectra to the AMES-Dusty-1999 models. The low gravity models are quite successful at reproducing (a) the $H$ bandpass profiles; (b) the weakness of CO absorption in the $K$ bandpass spectra; and (c) the absence of $\mathrm{Na} I$ absorption at $2.21 \mu \mathrm{m}$, though with stringent limits in only one source. There is an excellent correlation between independently derived spectroscopic temperatures and photometric temperatures, with a relatively small systematic offset of $250 \mathrm{~K}$.

Spectra of 2 planetary mass candidates and 3 very low mass brown dwarfs $\left(\mathrm{M} \leq 0.016 \mathrm{M}_{\odot}\right.$ at $\left.1 \mathrm{Myr}\right)$ show very strong water absorption consistent with L-type spectra, and have lower spectroscopic temperatures than the higher mass brown dwarfs, which is also predicted by the evolutionary tracks. The late spectral types confirm that these are cluster members, since they are too luminous to be background field dwarfs and the modest extinction seen in most cases demonstrates that they are not foreground dwarfs. We would expect many of the L-type sources to show Mtype spectra at optical wavelengths, since water absorption is deeper in low gravity dwarfs than high gravity dwarfs, according to the AMES-Dusty-1999 spectra. The fitted temperatures are higher than those usually associated with Ltype sources. The trend of CO absorption to be significantly weaker at low gravities, for effective temperatures in the range $2100-2900 \mathrm{~K}$, is a potentially powerful tool for identifying very young brown dwarfs, provided there is an independent means of measuring the effective temperature. This trend has been observed before in low gravity giant and supergiant stars (eg. Origlia, Moorwood \& Oliva) at both $2.3 \mu \mathrm{m}$ and $1.62 \mu \mathrm{m}$.

While the ages of the individual sources cannot be precisely measured, it is very unlikely the 15 photometric planetary mass candidates could be much more massive sources with ages $>20 \mathrm{Myr}$, since statistically we would expect $<1$ such source in our dataset. There is also some evidence for large $K$ bandpass excesses in 2 of the planetary mass candidates, obtained by combining our $J H$ data with the $H K$ data of Hillenbrand \& Carpenter 2000; however this must be confirmed by a single deep $J H K$ survey.

\section{ACKNOWLEDGEMENTS}

We wish to thank the staff of UKIRT, which is operated by the Joint Astronomy Centre on behalf of the UK Particle Physics and Astronomy Research Council (PPARC). We are very grateful to Sandy Leggett for providing her spectroscopic data of field dwarfs electonically, permitting a detailed comparison between these sources and the our substellar candidates without the need to repeat all of the same observations. We are indebted to Isabelle Baraffe for providing self consistent theoretical isochrones, without which the interpretation of the photometry would be considerably less secure. We thank Hugh Jones, Antonio Chrysostomou and Suzanne Ramsay-Howat for helpful discussions and thank the referee for a useful report. We thank the UKIRT, Gemini and WHT Panels for the Allocation of Telescope Time for supporting this project and the University of Hawaii's Hokupa'a team and Gemini Observatory staff for carrying out Gemini observations for us. PWL is supported by PPARC via a Post Doctoral Fellowship at the University of Hertfordshire. 


\section{References}

Adams F.C. \& Fatuzzo M. 1996, ApJ 464, 256

Allard F., Hauschilt P.H., Alexander D.R., Starrfield S. 1997, Ann.Rev.A\&A 35, 137

Allard F., Hauschildt P.H., \& Schweitzer A. 2000, ApJ 539, 366

Allard F., Hauschildt P.H., \& Schwenke D. 2000, ApJ 540, 1005

Allard F., Hauschildt P.H., \& Alexander D.R., Tamanai A., \& Schweitzer, A. 2001, ApJ (submitted)

Bally J., O’Dell C.R., \& McCaughrean M.J. 2000 AJ, 119, 2919

Baraffe I., Chabrier G., Allard F., \& Hauschildt P.H. 1998, A\&A 337,403 (BCAH)

Bejar V.J.S., Zapatero-Osorio M.R., \& Rebolo R. 1999, ApJ 521,671

Briceno C., Hartmann L., Stauffer J., \& Martin E. 1998, AJ 115, 2074

Burrows A., Marley M., Hubbard W.B., Lunine J.I., Guillot T., Saumon D., Freedman R., Sudarsky D., \& Sharp C. 1997, ApJ, 491,856 (BM97)

Cardelli J.A., Clayton G.C., \& Mathis J.S. 1989, ApJ, 345,245

Carpenter J.M., Hillenbrand L.A., \& Skrutskie M.F. 2001, AJ (in press), astro-ph 0102446

Chabrier G., Baraffe I., Allard F. \& Hauschildt P. 2000, ApJ 542, L119

Chen H., Bally J., O’Dell C.R., McCaughrean M.J., Thompson R.L., Rieke M., Schneider G., \& Young E.T. 1998 ApJ 492 , L173

Comeron F., Rieke G.H., Burrows A., Rieke M.J. 1993, ApJ 416, 185

Comeron F., Rieke G.H., \& Rieke M.J. 1996, ApJ 473, 294

D'Antona F., \& Mazzitelli I. 1994, ApJS 90, 467

D'Antona F., \& Mazzitelli I. 1997, MmSAI 68,607

Delfosse X., Tinney C.G., Forveille T., Epchtein N., Borsenberger J., Fouque P., Kimeswenger S., \& Tiphene D. 1999, A\&A Supp. Series 135,41

Hauschildt P.H., Allard F., Baron E., Schweitzer A. 1999, ApJ 312. 377

Hillenbrand L.A. 1997, AJ 113,1733

Hillenbrand L.A., \& Hartmann L.W. 1998, ApJ 492, 540

Hillenbrand L.A. \& Carpenter J.M. 2000, ApJ 540, 236 (HC)

Johnstone D. \& Bally J. 1999, ApJ 510, L49

Jones H.R.A., Longmore A.J., Jameson R.F., \& Mountain C.M. 1994, MNRAS 267, 413

Kirkpatrick J.D., Reid I.N., Liebert J., Cutri R.M., Nelson B., Beichman C.A., Dahn C.C., Monet D.G., Gizis J.E., \& Skrutskie M.F. 1999, ApJ 519, 802

Kirkpatrick J.D., Reid I.N., Liebert J., Gizis J.E., Burgasser A.J., Monet D.G., Dahn C.C., Nelson B., \& Williams R.J. 2000, AJ 120,447

Lada C.J., Muench A.A., Haisch K.E., Lada E.A., Alves J.F., Tollestrup E.V. \& Willner S.P. 2000, AJ 120, 3162

Lada C.J., Lada E.A., Clemens D.P., \& Bally B. 1994, ApJ 429, 694

Lancon A. \& Rocca-Volmerange B. 1992, A\&A Supp. Series 96, 593

Leggett S.K. 1992, ApJS 82, 351

Leggett S.K., Allard F., Berriman G., Dahn C.C., \& Hauschildt P.H. 1996, ApJS 104, 117

Leggett S.K., Allard F., Dahn C., Hauschildt P.H., Kerr T.H., \& Rayner J. 2000, ApJ 535, 965

Leggett S.K., Allard F., Geballe T.R., Hauschildt P.H., \& Schweitzer A. 2001, ApJ 548, 908

Lucas P.W. \& Roche P.F. 1998, MNRAS 299, 699

Lucas P.W. \& Roche P.F. 2000, MNRAS 314, 858 (Paper I)

Luhman K.L., Liebert J. \&Rieke G.H. 1997, ApJ 489, L165

Luhman K.L., \& Rieke G.H. 1998, ApJ 497,354

Luhman K.L., Rieke G.H., Lada C.J., \& Lada E.J. 1998a, ApJ 508, 347

Luhman K.L., Briceno C., Rieke G.H. \& Hartmann, Lee 1998b, ApJ 493, 909

Luhman K.L., Rieke G.H., Young E.T., Cotera A.S., Chen H., Rieke M.J., Schneider G. \& Thompson R.I. 2000 ApJ 540, 1016

Martin E.L., Rebolo R., \& Zapatero-Osorio 1996, ApJ 469, 706

McCaughrean M., Zinnecker H., Rayner J., \& Stauffer J. 1995, in "The Bottom of the Main Sequence and Beyond", ed. C. Tinney, p209, (Berlin: Springer)

Najita J.R, Tiede G.P., \& Carr J.S. 2000, ApJ 541, 977

Oasa Y., Tamura M., \& Sugitani K. 1999, ApJ 526, 336

O'Dell C.R., \& Wen Z. 1994, ApJ 436, 194

O'Dell C.R., \& Wong K. 1996, AJ 111, 846

O'Dell C.R., \& Yusef-Zadeh F 2000, AJ, 120, 382

Origlia L., Moorwood A.F.M., \& Oliva A\&A 1993, 280, 536

Partridge H.,\& Schwenke D.W. 1997, J. Chem. Phys. 106, 4618

Reid I.N, Kirkpatrick J.D. Liebert J., Burrows A., Gizis J.E., Burgasser A., Dahn C.C., Monet D., Cutri R., Beichman C.A., Skrutskie M. 1999, ApJ 521, 613

Saumon D., Hubbard W.B., Burrows A., Guillot T., Lunine J.I., \& Chabrier G. 1996, ApJ 460, 993 
Silk J. 1977, ApJ 214, 152

Skrutskie M.F., Meyer M.R., Whalen D., \& Hamilton C. 1996, AJ 112, 2168

Storzer H. \& Hollenbach D. 1999, ApJ 515, 669

Swenson F.J., Faulkner J., Rogers F.J., \& Iglesias C.A. 1994, ApJ 425, 286

Tamura M., Itoh Y., Oasa Y., \& Nakajima T. 1998, Science 282, 1095

Tatematsu K., Umemoto T., Kameya O., Hirano N., Hasegawa T., Hayashi M., Iwata T., Kaifu N., Mikamic H., Murata Y., Nakano M., Nakano T., Ohashi N., Sunada K., Takaba H., \& Yamamoto, S. 1993, ApJ 404, 643

Tinney C.G. 1993, ApJ 414, 279

Tokunaga A.T. \& Kobayashi N. 1999, AJ 117, 1010

Tokunaga A.T. 2000, in Allen's Astrophysical Quantities; ed. A.Cox, AIP Press, New York

van Altena W.F., Lee J.T., \& Hoffleit E.D., 1995, The General Catalogue of Trigonometric Parallaxes (New Haven: Yale O Univ. Obs.)

Wainscoat R.J., Cohen M., Volk K., Walker H., \& Schwartz D.E. 1992, ApJS 83,111

Ward-Thompson D., Scott P.F., Hills R.E., \& Andre P. 1994, MNRAS 268, 276

Wilking B.A., Greene T.P., \& Meyer M.R. 1999, AJ 117, 469

Zapatero Osorio M.R., Bejar V.J.S., Rebolo R., Martin E.L., \& Basri G. 1999, ApJ 524, L115

Zapatero Osorio M.R., Bejar V.J.S., Martin E.L., Rebolo R., Barrado y Navascues D., Bailer-Jones C.A.L., \& Mundt R. 2000, Science 290, 103 
Table 1 - Log of Spectroscopic Observations

\begin{tabular}{|c|c|c|c|c|c|c|c|}
\hline \multirow[t]{2}{*}{ Source } & \multirow[t]{2}{*}{$H^{a}$} & \multirow[t]{2}{*}{$\mathrm{A}(\mathrm{V})$} & \multirow[t]{2}{*}{$\operatorname{Mass}^{b}\left(\mathrm{M}_{\odot}\right)$} & \multicolumn{2}{|c|}{ Exposure (s) } & \multirow[t]{2}{*}{ Paired with } & \multirow[t]{2}{*}{ Source Notes } \\
\hline & & & & $H$ band & $K$ band & & \\
\hline $095-100$ & 13.25 & 0.0 & 0.090 & 2700 & - & $084-104$ & \\
\hline 019-108 & 15.08 & 8.6 & 0.090 & 5376 & 5376 & $023-115$ & \\
\hline $055-231$ & 13.27 & 0.0 & 0.080 & 960 & 640 & $069-210$ & $25 \mathrm{~nm}$ resolution, $K$ signal reduced by cloud \\
\hline $069-210$ & 13.67 & 0.0 & 0.074 & 960 & 640 & $055-231$ & $25 \mathrm{~nm}$ resolution, $K$ signal reduced by cloud \\
\hline $068-020$ & 13.83 & 1.0 & 0.073 & 720 & 560 & 091-019 & \\
\hline $053-503$ & 13.57 & 0.0 & 0.073 & 1080 & 1080 & $047-436$ & \\
\hline 091-019 & 13.68 & 0.0 & 0.071 & 720 & 560 & $068-020$ & \\
\hline $019-354$ & 14.08 & 1.5 & 0.067 & 900 & 960 & $014-413$ & \\
\hline $066-433$ & 16.54 & 12.6 & 0.066 & 5376 & - & $061-401$ & \\
\hline $043-014$ & 13.92 & 0.0 & 0.061 & 896 & - & - & \\
\hline $047-436$ & 14.39 & $5.5 ?$ & $0.06 ?$ & 1080 & 1080 & $053-503$ & anomalously blue (I-J) colour \\
\hline $014-413$ & 14.44 & 1.3 & 0.056 & 900 & 960 & $019-354$ & \\
\hline 013-306 & 15.61 & 6.4 & 0.056 & 3584 & - & 016-309 & \\
\hline 096-1944 & 14.96 & 0.8 & 0.042 & 2240 & 1792 & 084-1939 & \\
\hline 084-1939 & 15.15 & 0.6 & 0.034 & 2240 & 1792 & 096-1944 & \\
\hline 084-104 & 16.89 & 0.0 & 0.016 & 2700 & - & 095-100 & \\
\hline 016-319 & 17.26 & 1.9 & 0.016 & 3584 & - & 013-306 & \\
\hline $038-627$ & 17.71 & 2.2 & 0.014 & 3584 & - & & \\
\hline $061-401$ & 17.71 & 0.0 & 0.011 & 5376 & - & $066-433$ & \\
\hline 023-115 & 18.41 & 2.1 & 0.008 & 5376 & 5376 & 019-108 & $K$ spectrum corrupted by nebulosity \\
\hline $017-410$ & 12.23 & 1.2 & 0.30 & 900 & 960 & $014-413$ & serendipitous in slit \\
\hline $073-205$ & 15.94 & 11.8 & 0.080 & 960 & - & $055-231$ & serendipitous in slit \\
\hline $178-232$ & 12.17 & $14.5 ?$ & $0.4 ?$ & 240 & - & - & proplyd, higher mass \\
\hline Gl406 & 6.57 & 0.0 & - & - & 24 & - & M6V dwarf \\
\hline BRI0021-0214 & 11.85 & 0.0 & - & 960 & 480 & - & M9.5V dwarf, $25 \mathrm{~nm}$ resolution at $H$. \\
\hline DBD0205-1159 & 13.59 & 0.0 & - & 720 & 720 & - & L7V dwarf \\
\hline
\end{tabular}

Notes:

(a) $H$ magnitudes are in the UKIRT - UFTI system.

(b) The quoted masses are derived from the BM97 tracks, assuming an age of 1 Myr.

Table $2-\mathrm{H}_{2}$ emission in 047-436

Measured $\lambda(\mu \mathrm{m})$
1.945
1.956
2.032
2.122
2.202
2.223
2.249
2.345
2.407
2.423
2.453
2.474

$\begin{array}{lc}\text { Line } & \text { Equivalent Width }(\AA) \\ (2-1) \mathrm{S}(5) & 5 \pm 2 \\ (1-0) \mathrm{S}(3) & 18 \pm 2 \\ (1-0) \mathrm{S}(2) & 4 \pm 2 \\ (1-0) \mathrm{S}(1) & 15 \pm 2 \\ \mathrm{~S}(3),(4-3) \mathrm{S}(5) & 4 \pm 2 \\ (1-0) \mathrm{S}(0) & 5 \pm 2 \\ (2-1) \mathrm{S}(1) & 4 \pm 2 \\ (4-3) \mathrm{S}(3) ? & 5 \pm 2 \\ (1-0) \mathrm{Q}(1) & 15 \pm 3 \\ (1-0) \mathrm{Q}(3) & 9 \pm 3 \\ (1-0) \mathrm{Q}(5) & 9 \pm 3 \\ (1-0) \mathrm{Q}(6) & 7 \pm 3\end{array}$


Table 3 - Spectral Types and Temperatures

\begin{tabular}{|c|c|c|c|c|c|c|c|c|}
\hline \multirow[t]{2}{*}{ Source $^{a}$} & \multicolumn{2}{|c|}{ Water Indices ${ }^{b}$} & \multicolumn{2}{|c|}{$\operatorname{Teff}(K)^{c}$} & \multicolumn{2}{|c|}{ Sp.Type ${ }^{d}$} & \multirow[t]{2}{*}{ Mass $\left(\mathrm{M}_{\odot}\right)$} & \multirow[t]{2}{*}{ Source Notes } \\
\hline & $\mathrm{W}$ & $\mathrm{Q}$ & {$[\mathrm{g}]=3.5$} & {$[\mathrm{~g}]=4.0$} & $\mathrm{~W}$ & Q & & \\
\hline 095-100 & $0.667 \pm .011$ & $0.558 \pm .018$ & 2950 & 2750 & L0 & L1 & 0.090 & \\
\hline 019-108 & $0.998 \pm .008$ & $0.936 \pm .015$ & 4850 & 4900 & $<\mathrm{M} 1$ & $<\mathrm{M} 1$ & 0.090 & high $\mathrm{A}(\mathrm{V})$, v. young source or background star \\
\hline $055-231$ & $0.585 \pm .013$ & $0.479 \pm .015$ & 2600 & 2550 & L3 & $\mathrm{L} 4$ & 0.080 & \\
\hline 069-210 & $0.724 \pm .017$ & $0.597 \pm .031$ & 3400 & 2950 & M7 & M9 & 0.074 & \\
\hline 068-020 & $0.734 \pm .027$ & $0.555 \pm .021$ & 3450 & 3000 & M7 & L1 & 0.073 & \\
\hline 053-503 & $0.671 \pm .040$ & $0.631 \pm .044$ & 3050 & 2750 & L0 & M7 & 0.073 & \\
\hline 091-019 & $0.776 \pm .023$ & $0.615 \pm .024$ & 3550 & 3200 & M5 & M8 & 0.071 & \\
\hline 019-354 & $0.717 \pm .013$ & $0.596 \pm .014$ & 3400 & 2900 & M8 & M9 & 0.067 & \\
\hline $066-433$ & $0.945 \pm .051$ & $0.937 \pm .066$ & 4450 & 4500 & $<\mathrm{M} 1$ & $<\mathrm{M} 1$ & 0.066 & high $\mathrm{A}(\mathrm{V})$, v. young source or background star \\
\hline 043-014 & $0.631 \pm .044$ & $0.545 \pm .038$ & 2700 & 2650 & L1 & L1 & 0.061 & \\
\hline $047-436$ & $0.785 \pm .019$ & $0.921 \pm .047$ & - & - & - & - & $0.06 ?$ & $\mathrm{H}_{2}$ emission source \\
\hline $014-413$ & $0.672 \pm .014$ & $0.615 \pm .043$ & 3100 & 2750 & M9 & M8 & 0.056 & \\
\hline 013-306 & $0.618 \pm .031$ & $0.594 \pm .018$ & 2650 & 2600 & L2 & M9 & 0.056 & \\
\hline 096-1944 & $0.632 \pm .031$ & $0.562 \pm .022$ & 2700 & 2650 & L1 & L0 & 0.042 & \\
\hline 084-1939 & $0.581 \pm .050$ & $0.455 \pm .035$ & 2550 & 2550 & L3 & L5 & 0.034 & \\
\hline 084-104 & $0.471 \pm .066$ & $0.396 \pm .091$ & 2350 & 2350 & L8 & L8 & 0.016 & \\
\hline 016-319 & $0.359 \pm .094$ & $0.394 \pm .110$ & 2150 & 2100 & $>\mathrm{L} 8$ & L8 & 0.016 & poor sky subtraction? \\
\hline $038-627$ & $0.601 \pm .072$ & $0.453 \pm .044$ & 2600 & 2600 & L3 & L5 & 0.014 & \\
\hline 061-401 & $0.552 \pm .054$ & $0.480 \pm .085$ & 2500 & 2500 & L5 & $\mathrm{L} 4$ & 0.011 & \\
\hline 023-115 & - & $0.582 \pm .150$ & & - & - & L0 & 0.008 & \\
\hline 017-410 & $0.851 \pm .047$ & $0.617 \pm .030$ & 3850 & 3900 & $\sim \mathrm{M} 0$ & L2 & 0.30 & higher mass, unusual profile \\
\hline 073-205 & $0.693 \pm .280$ & $0.469 \pm .082$ & 3300 & 2800 & M9 & L5 & 0.080 & uncertain $\mathrm{W}$ index due to high $\mathrm{A}(\mathrm{V})$ \\
\hline $178-232$ & - & $0.834 \pm .017$ & - & - & - & & $0.4 ?$ & higher mass, proplyd, $\mathrm{A}(\mathrm{V})$ uncertain \\
\hline
\end{tabular}

Notes:

(a) The 3 sources below the line are not in the primary sample, see Section 3.1.

(b) The $\mathrm{W}$, and $\mathrm{Q}$ water indices are defined in the text. The quoted errors are based on apparent noise and do not include uncertainties in dereddening or the background subtraction.

(c) Effective Temperatures are derived from the best fit of the AMES-Dusty-1999 spectra to the 1.45-1.7 $\mu \mathrm{m}$ data at two different gravities.

(d) Spectral types are derived from the $\mathrm{W}$ and $\mathrm{Q}$ indices, calibrated by field dwarfs from Leggett et al.(2001)

Table $4-\mathrm{CO}$ v=2-0 detections

\begin{tabular}{lcc} 
Source & $\mathrm{K}_{C O}$ & CO Detection \\
\hline $053-503$ & $0.095 \pm 0.005$ & yes \\
$014-413$ & $0.097 \pm 0.030$ & yes \\
$096-1944$ & $0.059 \pm 0.035$ & yes \\
$055-231$ & $0.119 \pm 0.055$ & yes \\
$068-020$ & $0.063 \pm 0.030$ & $?$ \\
$084-1939$ & $0.032 \pm 0.047$ & no \\
$091-019$ & $0.023 \pm 0.053$ & no \\
$047-436$ & $0.034 \pm 0.026$ & no \\
$019-108$ & $0.039 \pm 0.080$ & no \\
$069-210$ & $0.153 \pm 0.082$ & no \\
$019-354$ & $0.058 \pm 0.040$ & no \\
\hline DBD0205-1159 & $0.196 \pm 0.025$ & yes \\
BRI0021-0214 & $0.154 \pm 0.007$ & yes \\
G1406 & $0.102 \pm 0.007$ & yes
\end{tabular}

Note: Sources with no CO detection typically have $\mathrm{K}_{C O}=0.02-0.03$ due to the wing of the $\mathrm{H}_{2} \mathrm{O}$ absorption band $\mathrm{H}_{2} \mathrm{O}$ centred at $2.7 \mu \mathrm{m}$. 
Table 5 - $K$ bandpass excesses in Planetary Mass Candidates

\begin{tabular}{ccccccccc} 
Source & $J$ & $H_{L R}^{a}$ & $H_{H C}^{a}$ & $K$ & $\mathrm{~A}(\mathrm{~V})^{b}$ & $\mathrm{E}(\mathrm{H}-\mathrm{K})^{c}$ & $\Delta H^{d}$ & $K$ band excess \\
\hline $115-106$ & 19.32 & 18.41 & 18.30 & 17.11 & 2.49 & 0.72 & 0.11 & yes \\
$076-147$ & 18.67 & 17.94 & 17.93 & 16.98 & 1.11 & 0.49 & 0.01 & yes \\
$084-322$ & 18.74 & 17.91 & 18.04 & 17.40 & 1.90 & -0.02 & 0.13 & no \\
\hline $066-229$ & 19.97 & 18.84 & 18.15 & 17.59 & 4.31 & $?$ & 0.69 & $?$ \\
$137-531$ & 18.89 & 17.90 & 18.32 & 17.28 & 3.38 & $?$ & 0.42 & $?$ \\
$092-531$ & 18.72 & 17.91 & 18.46 & 17.29 & 1.78 & $?$ & 0.55 & $?$
\end{tabular}

Notes:

(a) $H_{L R}$ and $H_{H C}$ are the $H$ bandpass fluxes measured in Paper I and HC respectively.

(b) Visual extinctions are derived from $(J-H)$ colours, with uncertainty of at least 1 mag.

(c) An intrinsic colour of $(H-K)=0.38$ is assumed for the photospheres.

(d) $\Delta H$ is the discrepancy between the Paper I and HC $H$ bandpass fluxes. 